\title{
Smooth interpolation between thermal Born and LPM
} rates

\section{J. Ghiglieri ${ }^{a}$ and M. Laine ${ }^{b, c}$}

${ }^{a}$ SUBATECH, Université de Nantes, IMT Atlantique, IN2P3/CNRS,

4 rue Alfred Kastler, La Chantrerie BP 20722, 44307 Nantes, France

${ }^{b} A E C$, Institute for Theoretical Physics, University of Bern,

Sidlerstrasse 5, CH-3012 Bern, Switzerland

${ }^{c}$ Department of Physics, University of Jyväskylä,

P.O. Box 35 (YFL), FI-40014, Finland

E-mail: jacopo.ghiglieri@subatech.in2p3.fr, laine@itp.unibe.ch

ABSTRACT: In a weakly coupled ultrarelativistic plasma, $1+n \leftrightarrow 2+n$ scatterings, with $n \geq 0$, need sometimes to be summed to all orders, in order to determine a leading-order interaction rate. To implement this "LPM resummation", kinematic approximations are invoked. However, in cosmological settings, where the temperature changes by many orders of magnitude and both small and large momenta may play a role, such approximations are not always justified. We suggest a procedure to smoothly interpolate between LPMresummed $1+n \leftrightarrow 2+n$ and Born-level $1 \leftrightarrow 2$ results, rendering the outcome applicable to a broader range of masses and momenta. The procedure is illustrated for right-handed neutrino production from a Standard Model plasma, and dilepton production from a QCD plasma.

KEYwords: Thermal Field Theory, Cosmology of Theories beyond the SM, Quark-Gluon Plasma

ArXiv EPrint: 2110.07149 


\section{Contents}

1 Introduction 1

2 Phase space for thermal $1 \leftrightarrow 2$ processes 2

2.1 General derivation in light-cone coordinates 2

$\begin{array}{ll}2.2 & \text { Examples of matrix elements squared }\end{array}$

3 Leading-order LPM resummation $\quad 7$

$\begin{array}{ll}3.1 & \text { Known implementations }\end{array}$

$\begin{array}{llr}3.2 & \text { Determination of matrix elements squared } & 9\end{array}$

$\begin{array}{lll}4 & \text { Interpolation } & 11\end{array}$

5 Crosscheck of UV asymptotics 13

6 Matching of IR divergences $\quad 15$

$\begin{array}{lll}7 & \text { Numerical evaluation } & 18\end{array}$

8 Conclusions and outlook $\quad 19$

\section{Introduction}

Many problems in particle cosmology and heavy ion collision physics involve the computation of thermal interaction rates. On one hand this comes about in the context of production rates, for instance of "freeze-in" dark matter candidates, gravitational waves, right-handed neutrinos influencing leptogenesis, or photons and dileptons in the collider setting. On the other hand interaction rates dictate how efficiently probes approach equilibrium or keep up with it, as is relevant for instance for "freeze-out" dark matter candidates, active neutrinos, or for the quenching of an energetic jet produced in an initial hard scattering or decay.

In terms of Feynman diagrams, the lowest-order topologies yielding an interaction rate are $1 \leftrightarrow 2$ processes, dubbed decays and inverse decays. However, the phase space available for these reactions is strongly constrained, implying that the corresponding interaction rate is proportional to a positive power of particle masses. If we go to the so-called ultrarelativistic (UR) regime, where all masses are small compared with the temperature $T$, then the rate of $1 \leftrightarrow 2$ processes gets suppressed compared with the scaling dimension $T$.

If a process is phase-space suppressed, it can experience a large correction by a soft additional scattering, which modifies the kinematics. Therefore $1+n \leftrightarrow 2+n$ processes, with $n \geq 0$, need to be considered, and in some cases summed to all orders. The corresponding physics is related to that governing the propagation of high-energy cosmic rays through the atmosphere, whereby the treatment goes under the name of Landau-Pomeranchuk-Migdal (LPM) resummation (cf., e.g., refs. [1-4] and references therein). An incomplete list of recent cosmological applications of LPM resummation can be found in refs. [4-13]. 
It is typical of resummations that their consistent implementation requires the presence of a scale hierarchy. In the case of LPM resummation, the scale hierarchy is that characterizing the UR regime, i.e. with masses small compared with momenta, the latter of which are of order $\pi T$. However, in cosmology, the temperature changes, so a given particle can be ultrarelativistic at early and non-relativistic at late times. Moreover, we are often interested in the overall abundance of a certain species, so that all momenta are integrated over. In these situations, the scale hierarchy justifying the LPM resummation gets compromised. In previous studies, the issue has been circumvented by somewhat ad hoc interpolations between LPM-resummed and Born-level computations [7, 9]. Recipes for switching off LPM resummation for large virtualities have been introduced for the dilepton case as well $[14,15]$. The goal of the present paper is to suggest how the interpolation can be implemented on the integrand level, rendering it smooth and numerically robust.

We start by working out the kinematics of Born-level $1 \leftrightarrow 2$ processes in a special coordinate system which permits us to put the expression in a form similar to that appearing in LPM resummation (cf. section 2). After recalling typical implementations of LPM resummation (cf. section 3), it is then straightforward to suggest an interpolation (cf. section 4). As consistency checks, we discuss how the result compares with well-known ultraviolet (UV) asymptotics at large virtualities (cf. section 5), and verify that it correctly accounts for infrared (IR) divergences (cf. section 6). After illustrating the interpolation numerically (cf. section 7), we summarize the recipe, and suggest how it can be extended to include specific NLO corrections (cf. section 8).

\section{Phase space for thermal $1 \leftrightarrow 2$ processes}

\subsection{General derivation in light-cone coordinates}

We start by recalling the derivation of the phase space average for thermal $1 \leftrightarrow 2$ reactions at the Born level, arriving at an expression (cf. eq. (2.25)) which can subsequently be interpolated (cf. section 4) to the result obtained from LPM resummation (cf. section 3). The derivation may look complicated and requires a few opportune choices of variables, but this is necessary for establishing eq. (2.25) in its desired form.

For determining the interaction rate originating from $1 \leftrightarrow 2$ reactions at the Born level (this is generically denoted by $\Gamma_{1 \leftrightarrow 2}^{\text {Born }}$, remarking that an overall normalization factor is needed for obtaining the physical rate, cf. footnotes 2 and 3), it is sufficient to compute the functional form originating from $1 \rightarrow 2$ decays of a would-be non-equilibrium particle. The four-momentum of the non-equilibrium particle is denoted by $\mathcal{K} \equiv(\omega, \mathbf{k})$. The corresponding matrix element squared, or "splitting function", summed over the spins and degeneracies of the final-state particles, reads $\Theta\left(\mathcal{P}_{a}, \mathcal{P}_{b}\right)$. Here $\mathcal{P}_{a, b}$ are the four-momenta of particles of types $a$ and $b$, both of which are assumed thermalized. Then the full rate reads

$$
\begin{aligned}
\Gamma_{1 \leftrightarrow 2}^{\text {Born }}= & \operatorname{scat}_{1 \rightarrow 2}(a, b) \Theta\left(\mathcal{P}_{a}, \mathcal{P}_{b}\right) \\
& +\operatorname{scat}_{2 \rightarrow 1}(-a ; b) \Theta\left(-\mathcal{P}_{a}, \mathcal{P}_{b}\right) \\
& +\operatorname{scat}_{2 \rightarrow 1}(-b ; a) \Theta\left(\mathcal{P}_{a},-\mathcal{P}_{b}\right)
\end{aligned}
$$


where we have defined thermal phase space averages as

$$
\begin{aligned}
\operatorname{scat}_{1 \rightarrow 2}(a, b) & \equiv \frac{1}{2} \int \mathrm{d} \Omega_{1 \rightarrow 2} \mathcal{N}_{a, b}, \\
\mathrm{~d} \Omega_{1 \rightarrow 2} & \equiv \frac{1}{(2 \pi)^{6}} \frac{\mathrm{d}^{3} \mathbf{p}_{a}}{2 \epsilon_{a}} \frac{\mathrm{d}^{3} \mathbf{p}_{b}}{2 \epsilon_{b}}(2 \pi)^{4} \delta^{(4)}\left(\mathcal{K}-\mathcal{P}_{a}-\mathcal{P}_{b}\right) \\
\mathcal{N}_{a, b} & \equiv \bar{n}_{\sigma_{a}}\left(\epsilon_{a}-\mu_{a}\right) \bar{n}_{\sigma_{b}}\left(\epsilon_{b}-\mu_{b}\right)-n_{\sigma_{a}}\left(\epsilon_{a}-\mu_{a}\right) n_{\sigma_{b}}\left(\epsilon_{b}-\mu_{b}\right), \\
\operatorname{scat}_{2 \rightarrow 1}(-a ; b) & \equiv \frac{1}{2} \int \mathrm{d} \Omega_{2 \rightarrow 1} \mathcal{N}_{a ; b}, \\
\mathrm{~d} \Omega_{2 \rightarrow 1} & \equiv \frac{1}{(2 \pi)^{6}} \frac{\mathrm{d}^{3} \mathbf{p}_{a}}{2 \epsilon_{a}} \frac{\mathrm{d}^{3} \mathbf{p}_{b}}{2 \epsilon_{b}}(2 \pi)^{4} \delta^{(4)}\left(\mathcal{K}+\mathcal{P}_{a}-\mathcal{P}_{b}\right), \\
\mathcal{N}_{a ; b} & \equiv n_{\sigma_{a}}\left(\epsilon_{a}+\mu_{a}\right) \bar{n}_{\sigma_{b}}\left(\epsilon_{b}-\mu_{b}\right)-\bar{n}_{\sigma_{a}}\left(\epsilon_{a}+\mu_{a}\right) n_{\sigma_{b}}\left(\epsilon_{b}-\mu_{b}\right)
\end{aligned}
$$

Here $\sigma=+(-)$ labels bosons (fermions), and the corresponding distribution functions are

$$
\bar{n}_{\sigma}(\epsilon) \equiv 1+n_{\sigma}(\epsilon), \quad n_{\sigma}(\epsilon) \equiv \frac{\sigma}{e^{\epsilon / T}-\sigma},
$$

where $T$ is the temperature. The overall factor $\frac{1}{2}$ in eqs. (2.2) and (2.5) is a convention, guaranteeing that $\Theta$ can be interpreted as the matrix element squared $\sum|\mathcal{M}|^{2}$ of a Boltzmann equation, ${ }^{1}$ though a division by $\omega$ is still needed for obtaining the rate proper.

Denoting the masses of the various particles by $M^{2} \equiv \mathcal{K}^{2} \equiv \omega^{2}-k^{2}, m_{a}^{2} \equiv \mathcal{P}_{a}^{2}$, the energy-momentum conservation constraints in eqs. (2.3) and (2.6) imply that the three channels in eq. (2.1) get realized if

$$
\begin{array}{ll}
\epsilon_{b}=\omega-\epsilon_{a}, & M>m_{a}+m_{b}, \\
\epsilon_{b}=\omega+\epsilon_{a}, & m_{b}>m_{a}+M, \\
\epsilon_{b}=\epsilon_{a}-\omega, & m_{a}>m_{b}+M,
\end{array}
$$

respectively. The goal is to integrate over $\mathbf{p}_{b}$ in eqs. (2.2) and (2.5), and then to combine the channels into a single expression, with the remaining average given by an integral over $\epsilon_{a}$.

As a first step, making use of $n(-x)=-\bar{n}(x)$, indicating the energy arguments of $\mathcal{N}_{a, b}$ explicitly, and carrying out the integral over $\mathbf{p}_{b}$, we can rewrite eq. (2.1) as

$$
\begin{aligned}
& \Gamma_{1 \leftrightarrow 2}^{\mathrm{Born}}=\int \frac{\mathrm{d}^{3} \mathbf{p}_{a}}{2(4 \pi)^{2}}\{ \\
& +\frac{\mathcal{N}_{a, b}\left(\epsilon_{a}-\mu_{a}, \omega-\epsilon_{a}-\mu_{b}\right)}{\epsilon_{a} \epsilon_{b\left(\mathbf{k}-\mathbf{p}_{a}\right)}}\left[\delta\left(\omega-\epsilon_{a}-\epsilon_{b\left(\mathbf{k}-\mathbf{p}_{a}\right)}\right)-\delta\left(\omega-\epsilon_{a}+\epsilon_{b\left(\mathbf{k}-\mathbf{p}_{a}\right)}\right)\right] \Theta\left(\mathcal{P}_{a}, \mathcal{K}-\mathcal{P}_{a}\right) \\
& \left.+\frac{\mathcal{N}_{a, b}\left(-\epsilon_{a}-\mu_{a}, \omega+\epsilon_{a}-\mu_{b}\right)}{\epsilon_{a} \epsilon_{b\left(\mathbf{k}+\mathbf{p}_{a}\right)}}\left[-\delta\left(\omega+\epsilon_{a}-\epsilon_{b\left(\mathbf{k}+\mathbf{p}_{a}\right)}\right)\right] \Theta\left(-\mathcal{P}_{a}, \mathcal{K}+\mathcal{P}_{a}\right)\right\}
\end{aligned}
$$

\footnotetext{
${ }^{1}$ In case of identical final-state particles, this should be replaced by $\frac{1}{2} \sum|\mathcal{M}|^{2}$ as usual.
} 
The Dirac- $\delta$ constraints can be combined into

$$
\begin{aligned}
& \Gamma_{1 \leftrightarrow 2}^{\text {Born }}=\int \frac{\mathrm{d}^{3} \mathbf{p}_{a}}{(4 \pi)^{2}}\{ \\
& +\frac{\mathcal{N}_{a, b}\left(\epsilon_{a}-\mu_{a}, \omega-\epsilon_{a}-\mu_{b}\right)}{\epsilon_{a}} \operatorname{sign}\left(\omega-\epsilon_{a}\right) \delta\left[\left(\omega-\epsilon_{a}\right)^{2}-\epsilon_{b\left(\mathbf{k}-\mathbf{p}_{a}\right)}^{2}\right] \Theta\left(\mathcal{P}_{a}, \mathcal{K}-\mathcal{P}_{a}\right) \\
& \left.\quad-\frac{\mathcal{N}_{a, b}\left(-\epsilon_{a}-\mu_{a}, \omega+\epsilon_{a}-\mu_{b}\right)}{\epsilon_{a}} \operatorname{sign}\left(\omega+\epsilon_{a}\right) \delta\left[\left(\omega+\epsilon_{a}\right)^{2}-\epsilon_{b\left(\mathbf{k}+\mathbf{p}_{a}\right)}^{2}\right] \Theta\left(-\mathcal{P}_{a}, \mathcal{K}+\mathcal{P}_{a}\right)\right\} .
\end{aligned}
$$

To carry out the integral over $\mathbf{p}_{a}$, one normally goes over to spherical coordinates, selecting $\mathbf{k}$ as the $z$-axis. However, in order to make contact with LPM resummation [1-4], we employ light-cone coordinates instead, writing

$$
\mathbf{p}_{a}=p_{a \|} \mathbf{e}_{\mathbf{k}}+\mathbf{p}_{\perp}, \quad \mathbf{e}_{\mathbf{k}} \equiv \frac{\mathbf{k}}{k} .
$$

Furthermore we substitute $\mathbf{p}_{a} \rightarrow-\mathbf{p}_{a}$ in the latter term of eq. (2.13). Then the energies of the particles of types $a$ and $b$ take the forms

$$
\epsilon_{a}=\sqrt{p_{a \|}^{2}+p_{\perp}^{2}+m_{a}^{2}}, \quad \epsilon_{b}=\sqrt{\left(k-p_{a \|}\right)^{2}+p_{\perp}^{2}+m_{b}^{2}}, \quad p_{\perp} \equiv\left|\mathbf{p}_{\perp}\right| .
$$

The arguments of the Dirac- $\delta$ 's from eq. (2.13) become

$$
\left(\omega \mp \epsilon_{a}\right)^{2}-\epsilon_{b\left(\mathbf{k}-\mathbf{p}_{a}\right)}^{2}=M^{2}+m_{a}^{2}-m_{b}^{2} \mp 2 \omega \epsilon_{a}+2 k p_{a \|},
$$

which when put to zero establish the relation of $p_{a \|}$ and $\epsilon_{a}$ (cf. eq. (2.22)).

Writing the integration measure as $\int \mathrm{d}^{3} \mathbf{p}_{a}=\int \mathrm{d}^{2} \mathbf{p}_{\perp} \int_{-\infty}^{\infty} \mathrm{d} p_{a \|}$, the sign of $p_{a \|}$ can be dealt with by a special representation of the integrand. Inside $\Theta$, we envisage that $p_{a \|}$ is solved for by setting eq. (2.16) to zero. The function $\Theta$ is thereby expressed as a function of $\epsilon_{a}$ and $\mathbf{p}_{\perp}$, where in turn $\epsilon_{a}$ is an even function of $p_{a \|}$, through eq. (2.15). We can then write

$$
\begin{aligned}
\int_{-\infty}^{\infty} \mathrm{d} p_{a \|} \delta\left(\Delta+2 k p_{a \|}\right) \phi\left(p_{a \|}^{2}\right) & =\int_{0}^{\infty} \mathrm{d} p_{a \|}\left[\delta\left(\Delta+2 k p_{a \|}\right)+\delta\left(\Delta-2 k p_{a \|}\right)\right] \phi\left(p_{a \|}^{2}\right) \\
& =\int_{0}^{\infty} \mathrm{d} p_{a \|} 4 k p_{a \|} \delta\left(\Delta^{2}-4 k^{2} p_{a \|}^{2}\right) \phi\left(p_{a \|}^{2}\right)
\end{aligned}
$$

Inserting this into eq. (2.13), and denoting $\int_{\mathbf{p}_{\perp}} \equiv \int \mathrm{d}^{2} \mathbf{p}_{\perp} /(2 \pi)^{2}$, we are faced with

$$
\begin{aligned}
\Gamma_{1 \leftrightarrow 2}^{\mathrm{Born}}= & \int_{\mathbf{p}_{\perp}} \int_{0}^{\infty} \mathrm{d} p_{a \|} p_{a \|} k\{ \\
& +\frac{\operatorname{sign}\left(\omega-\epsilon_{a}\right)\left[1+n_{\sigma_{a}}\left(\epsilon_{a}-\mu_{a}\right)+n_{\sigma_{b}}\left(\omega-\epsilon_{a}-\mu_{b}\right)\right]}{\epsilon_{a}} \\
& \times \delta\left[\left(M^{2}+m_{a}^{2}-m_{b}^{2}-2 \omega \epsilon_{a}\right)^{2}-4 k^{2} p_{a \|}^{2}\right] \Theta\left(\epsilon_{a}, \mathbf{p}_{a}, \omega-\epsilon_{a}, \mathbf{k}-\mathbf{p}_{a}\right) \\
& -\frac{\operatorname{sign}\left(\omega+\epsilon_{a}\right)\left[1+n_{\sigma_{a}}\left(-\epsilon_{a}-\mu_{a}\right)+n_{\sigma_{b}}\left(\omega+\epsilon_{a}-\mu_{b}\right)\right]}{\epsilon_{a}} \\
& \left.\times \delta\left[\left(M^{2}+m_{a}^{2}-m_{b}^{2}+2 \omega \epsilon_{a}\right)^{2}-4 k^{2} p_{a \|}^{2}\right] \Theta\left(-\epsilon_{a}, \mathbf{p}_{a}, \omega+\epsilon_{a}, \mathbf{k}-\mathbf{p}_{a}\right)\right\}
\end{aligned}
$$


Now, by making use of eq. (2.15), we can replace $\left(\mathbf{p}_{\perp}, p_{a \|}\right)$ as integration variables through $\left(\mathbf{p}_{\perp}, \epsilon_{a}\right)$. Then, we substitute $\epsilon_{a} \rightarrow-\epsilon_{a}$ in the second structure of eq. (2.18). The overall minus sign must be accounted for, whereby the integrand is weighted by $\operatorname{sign}\left(\epsilon_{a}\left(\omega-\epsilon_{a}\right)\right)$. Inside the Dirac- $\delta$, we set $p_{a \|}^{2}=\epsilon_{a}^{2}-p_{\perp}^{2}-m_{a}^{2}$. Finally, we pull out a common factor $8 k^{2} \epsilon_{a}\left(\omega-\epsilon_{a}\right) / \omega$ from the argument of the Dirac- $\delta$, whereby the weight function gets multiplied by $\frac{\omega}{8 k^{2}\left|\epsilon_{a}\left(\omega-\epsilon_{a}\right)\right|}$. Altogether this yields

$$
\begin{aligned}
\Gamma_{1 \leftrightarrow 2}^{\text {Born }}= & \frac{\omega}{8 k}\left\{\int_{-\infty}^{-m_{a}}+\int_{m_{a}}^{\infty}\right\} \frac{\mathrm{d} \epsilon_{a}}{\epsilon_{a}\left(\omega-\epsilon_{a}\right)} \\
& \times \int_{\left|\mathbf{p}_{\perp}\right|<\sqrt{\epsilon_{a}^{2}-m_{a}^{2}}}\left[1+n_{\sigma_{a}}\left(\epsilon_{a}-\mu_{a}\right)+n_{\sigma_{b}}\left(\omega-\epsilon_{a}-\mu_{b}\right)\right] \\
& \times \delta\left[\frac{p_{\perp}^{2}}{2 \epsilon_{a}}+\frac{p_{\perp}^{2}}{2\left(\omega-\epsilon_{a}\right)}+\frac{\omega M^{2}\left(\epsilon_{a}-\epsilon_{a}^{-}\right)\left(\epsilon_{a}-\epsilon_{a}^{+}\right)}{2 k^{2} \epsilon_{a}\left(\omega-\epsilon_{a}\right)}\right] \Theta\left(\mathcal{P}_{a}, \mathcal{K}-\mathcal{P}_{a}\right),
\end{aligned}
$$

where we have denoted

$$
\epsilon_{a}^{ \pm} \equiv \frac{\omega\left(M^{2}+m_{a}^{2}-m_{b}^{2}\right) \pm k \sqrt{\lambda}\left(M^{2}, m_{a}^{2}, m_{b}^{2}\right)}{2 M^{2}},
$$

with the Källén function given by

$$
\sqrt{\lambda}\left(M^{2}, m_{a}^{2}, m_{b}^{2}\right) \equiv \sqrt{M^{4}+m_{a}^{4}+m_{b}^{4}-2 M^{2}\left(m_{a}^{2}+m_{b}^{2}\right)-2 m_{a}^{2} m_{b}^{2}} .
$$

The longitudinal momentum components, appearing inside $\Theta$, satisfy eq. (2.16), viz.

$$
p_{a \|}=\left[\epsilon_{a}+\frac{m_{b}^{2}-m_{a}^{2}-M^{2}}{2 \omega}\right] \frac{\omega}{k}, \quad k-p_{a \|}=\left[\omega-\epsilon_{a}+\frac{m_{a}^{2}-m_{b}^{2}-M^{2}}{2 \omega}\right] \frac{\omega}{k} .
$$

As guaranteed by the Dirac- $\delta$ in eq. (2.19), $\epsilon_{a}$ and $p_{\perp}^{2}$ are not independent; their relation can also be expressed as

$$
\epsilon_{a}^{2}-p_{a \|}^{2}=p_{\perp}^{2}+m_{a}^{2}, \quad\left(\omega-\epsilon_{a}\right)^{2}-\left(k-p_{a \|}\right)^{2}=p_{\perp}^{2}+m_{b}^{2} .
$$

Inspecting the smallest and largest values of $p_{\perp}^{2}$ in eq. (2.19), the Dirac- $\delta$ gets realized if

$$
\left(\epsilon_{a}-\epsilon_{a}^{-}\right)\left(\epsilon_{a}-\epsilon_{a}^{+}\right)<0 \wedge \epsilon_{a}^{2}-m_{a}^{2}+\frac{M^{2}}{k^{2}}\left(\epsilon_{a}-\epsilon_{a}^{-}\right)\left(\epsilon_{a}-\epsilon_{a}^{+}\right)>0
$$

The latter condition can be completed into a square, and is thus always satisfied. This implies that actually no upper bound needs to be imposed on $p_{\perp}$. The former constraint sets the viable range as $\epsilon_{a}^{-}<\epsilon_{a}<\epsilon_{a}^{+}$. We also note that $\epsilon_{a}>m_{a}$ if $M>m_{a}+m_{b}$ or $m_{a}>M+m_{b}$, and $\epsilon_{a}<-m_{a}$ if $m_{b}>M+m_{a}$. The domain $-m_{a}<\epsilon_{a}<m_{a}$ gives no contribution, and does not need to be explicitly excluded in eq. (2.19). Making also an effort to write the argument of the Dirac- $\delta$ in a more transparent form, we thus end up with

$$
\begin{aligned}
\Gamma_{1 \leftrightarrow 2}^{\text {Born }}= & \frac{\omega}{8 k} \int_{-\infty}^{\infty} \frac{\mathrm{d} \epsilon_{a}}{\epsilon_{a}\left(\omega-\epsilon_{a}\right)}\left[1+n_{\sigma_{a}}\left(\epsilon_{a}-\mu_{a}\right)+n_{\sigma_{b}}\left(\omega-\epsilon_{a}-\mu_{b}\right)\right] \int_{\mathbf{p}_{\perp}} \Theta\left(\mathcal{P}_{a}, \mathcal{K}-\mathcal{P}_{a}\right) \\
& \times \delta\left[\frac{p_{\perp}^{2}+m_{a}^{2}+\frac{\left(m_{b}^{2}-m_{a}^{2}-M^{2}\right)^{2}}{4 k^{2}}}{2 \epsilon_{a}}+\frac{p_{\perp}^{2}+m_{b}^{2}+\frac{\left(m_{a}^{2}-m_{b}^{2}-M^{2}\right)^{2}}{4 k^{2}}}{2\left(\omega-\epsilon_{a}\right)}-\frac{\omega M^{2}}{2 k^{2}}\right] .
\end{aligned}
$$

For a polynomial $\Theta$, the remaining integrals could be carried out in terms of polylogarithms, however for us it is advantageous to leave them unintegrated. 


\subsection{Examples of matrix elements squared}

As a first example of $\Theta$ in eq. (2.25), consider the production of right-handed neutrinos from the symmetric phase of a Standard Model plasma [4]. In the $1 \leftrightarrow 2$ process, the Standard Model particles participating in this reaction are leptons $(\ell)$ and scalars $(\phi)$. Being massive, the right-handed neutrinos can be produced with positive or negative helicity $(\tau= \pm)$ [16]. The corresponding rate, modulo overall normalization, can be expressed as

$$
\Gamma_{1 \leftrightarrow 2}^{\operatorname{Born}(\tau)}=\operatorname{scat}_{1 \leftrightarrow 2}(\ell, \phi) \Theta^{\tau}\left(\mathcal{P}_{\ell}, \mathcal{P}_{\phi}\right) .
$$

For negative helicity, as is carried by massless Standard Model leptons, the rate is suppressed by the right-handed neutrino mass, viz. ${ }^{2}$

$$
\Theta^{-}=2(\omega-k)\left(\epsilon_{\ell}+p_{\ell \|}\right),
$$

where $p_{\ell \|}$ is given by eq. (2.22), and in the symmetric phase $m_{a}=m_{\ell}=0$.

Now, when we make contact with LPM resummation (cf. section 3), we need to consider $\Theta$ in the UR limit. This is defined by assuming that all particle masses are small compared with the momenta of the particles $\left(m_{i}^{2}, M^{2} \ll \epsilon_{i}^{2}, k^{2}\right)$. In the UR regime, $\omega \approx k+M^{2} /(2 k)$, and then eq. (2.22) implies that $p_{a \|} \approx \epsilon_{a}$. Therefore the negative helicity production rate can be estimated as $\Theta^{-} \stackrel{\mathrm{UR}}{\approx} 2 M^{2} \epsilon_{\ell} / \omega$, where we went back to "energy-like" variables in the end.

For positive helicity, the right-handed neutrinos are in their natural state, whereas the active leptons experience "chiral suppression". This can be lifted through angular momentum transfer, as is manifested by

$$
\Theta^{+}=2(\omega+k)\left(\epsilon_{\ell}-p_{\ell \|}\right)=\frac{2(\omega+k) p_{\perp}^{2}}{\epsilon_{\ell}+p_{\ell \|}},
$$

where we made use of eq. (2.23). In the UR regime, this reduces to $\Theta^{+} \stackrel{\mathrm{UR}}{\approx} 2 \omega p_{\perp}^{2} / \epsilon_{\ell}$.

As a second example, we consider photon or dilepton production from a QCD plasma [1-3]. Now the rate can be expressed as $^{3}$

$$
\begin{aligned}
\Gamma_{1 \leftrightarrow 2}^{\operatorname{Born}(\mu \nu)} & =\operatorname{scat}_{1 \leftrightarrow 2}(q, \bar{q}) \Theta^{\mu \nu}\left(\mathcal{P}_{q}, \mathcal{P}_{\bar{q}}\right), \\
\Theta^{\mu \nu}\left(\mathcal{P}_{q}, \mathcal{P}_{\bar{q}}\right) & =2 N_{\mathrm{c}}\left[2\left(\mathcal{P}_{q}^{\mu} \mathcal{P}_{\bar{q}}^{\nu}+\mathcal{P}_{q}^{\nu} \mathcal{P}_{\bar{q}}^{\mu}\right)-\eta^{\mu \nu} M^{2}\right],
\end{aligned}
$$

where $q$ denotes a (possibly massive) quark, $\bar{q}$ an antiquark, and $\eta^{\mu \nu} \equiv \operatorname{diag}(+---)$. For on-shell photon production, we are interested in the transverse projection

$$
\Gamma_{1 \leftrightarrow 2}^{\mathrm{Born}(\mathrm{T})} \equiv\left(\delta_{i j}-\frac{k_{i} k_{j}}{k^{2}}\right) \Gamma_{1 \leftrightarrow 2}^{\mathrm{Born}(i j)},
$$

\footnotetext{
${ }^{2}$ To be precise, the "rate" we consider here is $\operatorname{Im}\left[\bar{u}_{\mathbf{k} \tau} a_{\mathrm{L}} \Pi_{a}^{\mathrm{R}} a_{\mathrm{R}} u_{\mathbf{k} \tau}\right]$, where $\Pi_{a}^{\mathrm{R}}$ is the retarded correlator associated with the current $\tilde{\phi}^{\dagger} \ell_{a}$, and $a \in\{e, \mu, \tau\}$ is an active lepton flavour. The actual production or equilibration rate is obtained by multiplying this by $h_{\nu}^{2} / \omega$, where $h_{\nu}$ is a neutrino Yukawa coupling.

${ }^{3}$ To be precise, the "rate" we consider here is $\operatorname{Im}\left[\Pi^{\mathrm{R} \mu \nu}\right]$, where $\Pi^{\mathrm{R} \mu \nu}$ is the retarded correlator associated with the vector current $\bar{\psi} \gamma^{\mu} \psi$. The actual photon or dilepton production rate is obtained by multiplying this by a kinematic prefactor and by an appropriate power of electromagnetic couplings.
} 
whereas for dileptons the longitudinal components need to be added,

$$
\Gamma_{1 \leftrightarrow 2}^{\mathrm{Born}(\mathrm{L})} \equiv \frac{k_{i} k_{j}}{k^{2}} \Gamma_{1 \leftrightarrow 2}^{\mathrm{Born}(i j)}-\Gamma_{1 \leftrightarrow 2}^{\mathrm{Born}(00)}=\frac{M^{2}}{k^{2}} \Gamma_{1 \leftrightarrow 2}^{\mathrm{Born}(00)} .
$$

For the latter representation, we made use of the Ward identity $\mathcal{K}_{\mu} \Gamma_{1 \leftrightarrow 2}^{\mu \nu}=0$. The vector correlator is the sum of the transverse and longitudinal ones,

$$
\Gamma^{\mathrm{V}} \equiv \Gamma^{\mathrm{T}}+\Gamma^{\mathrm{L}}=\left(-\eta_{\mu \nu}\right) \Gamma^{(\mu \nu)} .
$$

Consider first the longitudinal polarization. Employing the latter representation in eq. (2.32), and inserting eq. (2.30), the weight function becomes

$$
\Theta^{\mathrm{L}}=\frac{2 N_{\mathrm{c}} M^{2}\left(4 \epsilon_{q} \epsilon_{\bar{q}}-M^{2}\right)}{k^{2}} .
$$

In the UR regime, this reads $\Theta^{\mathrm{L}} \stackrel{\mathrm{UR}}{\approx} 8 N_{\mathrm{c}} M^{2} \epsilon_{q} \epsilon_{\bar{q}} / \omega^{2}$, rendering a case similar to $\Theta^{-}$above.

For the transverse channel, recalling $\mathbf{p}_{b \perp}=-\mathbf{p}_{a \perp}$, eqs. (2.30) and (2.31) yield

$$
\Theta^{\mathrm{T}}=4 N_{\mathrm{c}}\left(-2 p_{\perp}^{2}+M^{2}\right)
$$

This case differs from those considered before, as $p_{\perp}^{2}$ appears. As dictated by eq. (2.25), at the Born level the magnitude of $p_{\perp}^{2}$ is related to the energies and masses. In particular, in the UR regime, $M^{2}$ and $p_{\perp}^{2}$ are of the same order. Then we can eliminate $M^{2}$ in favour of $p_{\perp}^{2}$ through the Dirac- $\delta$ constraint in eq. (2.25), which leads to the approximate form that often appears in literature, $\Theta^{\mathrm{T}} \stackrel{\mathrm{UR}}{\approx} 4 N_{\mathrm{c}} p_{\perp}^{2}\left[\epsilon_{q}^{2}+\left(\omega-\epsilon_{q}\right)^{2}\right] /\left[\epsilon_{q}\left(\omega-\epsilon_{q}\right)\right]$.

\section{Leading-order LPM resummation}

\subsection{Known implementations}

The goal now is to compare eq. (2.25) with the framework of leading-order LPM resummation. For the benefit of an impatient reader, we first reiterate known formulations, returning in section 3.2 to how the matrix elements squared appearing in them can be derived. All the while, it is important to keep in mind that as LPM resummation is viable for UR kinematics, there is latitude in how kinematic variables are chosen beyond this limit.

Starting with right-handed neutrinos [4], but resolving the helicity channels [16]; undoing the normalization by $\omega$ that is often invoked in the literature (cf. footnote 2); and making a few substitutions $k \rightarrow \omega$ to render eqs. (3.1), (3.3) close in appearance to eqs. (3.6), (3.7), we can re-express the LPM-resummed result as

$$
\begin{aligned}
\Gamma_{1+n \leftrightarrow 2+n}^{\mathrm{LPM}(\tau)}= & \frac{1}{8} \int_{-\infty}^{\infty} \frac{\mathrm{d} \epsilon_{\ell}}{\epsilon_{\ell}\left(\omega-\epsilon_{\ell}\right)}\left[1-n_{\mathrm{F}}\left(\epsilon_{\ell}-\mu_{\ell}\right)+n_{\mathrm{B}}\left(\omega-\epsilon_{\ell}-\mu_{\phi}\right)\right] \\
& \times \lim _{\mathbf{y}_{\perp} \rightarrow \mathbf{0}} \mathbb{P}\left\{\frac{2 M^{2} \epsilon_{\ell} \delta_{\tau,-}}{\omega} \frac{\operatorname{Im}\left[g\left(\mathbf{y}_{\perp}\right)\right]}{\pi}+\frac{2 \omega \delta_{\tau,+}}{\epsilon_{\ell}} \frac{\operatorname{Im}\left[\nabla_{\perp} \cdot \mathbf{f}\left(\mathbf{y}_{\perp}\right)\right]}{\pi}\right\},
\end{aligned}
$$


where $\mathbb{P}$ stands for a principal value, and $g$ and $\mathbf{f}$ are wave functions satisfying

$$
\begin{aligned}
& \left(\hat{H}-i 0^{+}\right) g\left(\mathbf{y}_{\perp}\right)=\delta^{(2)}\left(\mathbf{y}_{\perp}\right), \quad\left(\hat{H}-i 0^{+}\right) \mathbf{f}\left(\mathbf{y}_{\perp}\right)=-\nabla_{\perp} \delta^{(2)}\left(\mathbf{y}_{\perp}\right), \\
& \hat{H} \equiv \frac{\delta m_{\ell T}^{2}-\nabla_{\perp}^{2}}{2 \epsilon_{\ell}}+\frac{m_{\phi T}^{2}-\nabla_{\perp}^{2}}{2\left(\omega-\epsilon_{\ell}\right)}-\frac{M^{2}}{2 \omega}-i \sum_{i=1}^{2} g_{\mathrm{E} i}^{2} C_{i} \phi\left(m_{\mathrm{E} i} y_{\perp}\right) .
\end{aligned}
$$

Here $\delta m_{\ell T}^{2} \approx\left(g_{1}^{2} C_{1}+g_{2}^{2} C_{2}\right) T^{2} / 4$ is an "asymptotic" thermal lepton mass [17] (quadratic appearances of chemical potentials have been omitted), with $C_{1} \equiv 1 / 4, C_{2} \equiv 3 / 4 ; m_{\phi T}^{2} \approx$ $-m_{H}^{2} / 2+\left(g_{1}^{2}+3 g_{2}^{2}+4 h_{t}^{2}+8 \lambda\right) T^{2} / 16$ is a thermal Higgs mass [18]; and the thermal width accounts for soft gauge scatterings [19],

$$
\phi\left(m_{\mathrm{E}} y_{\perp}\right) \equiv \int_{\mathbf{q}_{\perp}}\left(1-e^{i \mathbf{q}_{\perp} \cdot \mathbf{y}_{\perp}}\right)\left(\frac{1}{q_{\perp}^{2}}-\frac{1}{q_{\perp}^{2}+m_{\mathrm{E}}^{2}}\right)=\frac{1}{2 \pi}\left[\ln \left(\frac{m_{\mathrm{E}} y_{\perp}}{2}\right)+\gamma_{\mathrm{E}}+K_{0}\left(m_{\mathrm{E}} y_{\perp}\right)\right]
$$

where $K_{0}$ is a modified Bessel function. The Debye masses

$$
m_{\mathrm{E} 1}^{2} \approx\left(\frac{n_{S}}{6}+\frac{5 n_{G}}{9}\right) g_{1}^{2} T^{2}, \quad m_{\mathrm{E} 2}^{2} \approx\left(\frac{2}{3}+\frac{n_{S}}{6}+\frac{n_{G}}{3}\right) g_{2}^{2} T^{2}, \quad n_{S} \equiv 1, \quad n_{G} \equiv 3,
$$

and the gauge couplings $g_{\mathrm{E} i}^{2} \approx g_{i}^{2} T$ are those of the dimensionally reduced effective theory [20].

We note from eqs. (3.3), (3.4) that the parametric magnitude of the thermal width is $\sim g_{2}^{2} T / \pi$. If $\delta m_{\ell T}^{2} / \epsilon_{\ell}, m_{\phi T}^{2} /\left(\omega-\epsilon_{\ell}\right)$ or $M^{2} / \omega$ is much larger than this, then the width can be omitted $\left(\phi \rightarrow 0^{+}\right)$. Then eq. (3.2) can be solved with Fourier transformations. Recalling the UR limits of $\Theta^{-}$and $\Theta^{+}$from below eqs. (2.27) and (2.28), respectively, we find that in this situation there is a perfect match between eq. (2.25) and eqs. (3.1), (3.3).

A similar exercise is possible for photons and dileptons produced from a massless QCD plasma. Following ref. [3], one viable representation, with $i=T, L$, reads

$$
\begin{aligned}
\Gamma_{1+n \leftrightarrow 2+n}^{\mathrm{LPM}(i)} \equiv & N_{\mathrm{c}} \int_{-\infty}^{\infty} \mathrm{d} \epsilon_{q}\left[1-n_{\mathrm{F}}\left(\epsilon_{q}-\mu_{q}\right)-n_{\mathrm{F}}\left(\omega-\epsilon_{q}+\mu_{q}\right)\right] \\
& \times \lim _{\mathbf{y}_{\perp} \rightarrow \mathbf{0}} \mathbb{P}\left\{\frac{M^{2} \delta_{i, L}}{\omega^{2}} \frac{\operatorname{Im}\left[g\left(\mathbf{y}_{\perp}\right)\right]}{\pi}+\frac{\left[\epsilon_{q}^{2}+\left(\omega-\epsilon_{q}\right)^{2}\right] \delta_{i, T}}{2 \epsilon_{q}^{2}\left(\omega-\epsilon_{q}\right)^{2}} \frac{\operatorname{Im}\left[\nabla_{\perp} \cdot \mathbf{f}\left(\mathbf{y}_{\perp}\right)\right]}{\pi}\right\},
\end{aligned}
$$

where $g$ and $\mathbf{f}$ are Green's functions in the sense of eq. (3.2). Given that the quark and antiquark are degenerate, the operator $\hat{H}$ can be simplified into

$$
\hat{H}=\frac{\omega\left(m_{\infty}^{2}-\nabla_{\perp}^{2}\right)}{2 \epsilon_{q}\left(\omega-\epsilon_{q}\right)}-\frac{M^{2}}{2 \omega}-i g_{\mathrm{E} 3}^{2} C_{3} \phi\left(m_{\mathrm{E} 3} y_{\perp}\right),
$$

where $m_{\infty}^{2} \equiv \delta m_{q T}^{2}=\delta m_{\bar{q} T}^{2} \approx g_{3}^{2} C_{3} T^{2} / 4$ is the asymptotic quark thermal mass; $C_{3} \equiv$ $\left(N_{\mathrm{c}}^{2}-1\right) /\left(2 N_{\mathrm{c}}\right)$; and $m_{\mathrm{E} 3}^{2} \approx\left(N_{\mathrm{c}} / 3+N_{\mathrm{f}} / 6\right) g_{3}^{2} T^{2}$ as well as $g_{\mathrm{E} 3}^{2} \approx g_{3}^{2} T$ are parameters of the dimensionally reduced theory $[20]$.

Once again, eqs. (3.6) and (3.7) agree with eq. (2.25) in the limit $M^{2} / \omega, m_{\infty}^{2} / \epsilon_{q} \gg$ $g_{3}^{2} T / \pi$, if we make use of the UR limits for the two polarization states, as given below eqs. (2.34) and (2.35), respectively. 


\subsection{Determination of matrix elements squared}

We now return to how the matrix elements squared, visible on the second rows of eqs. (3.1) and (3.6), can be derived from the UR limit of the Hard Thermal Loop (HTL) effective theory. Like in eq. (2.1), it is sufficient to consider a $1 \rightarrow 2$ decay, with the other channels given by crossings, which are automatically incorporated in the coordinate system of eq. (2.25).

To obtain these contributions, the resummed scalar and fermion propagators are needed. The scalar propagator is simple, as thermal corrections modify the mass but not the structure. Then the spectral function (imaginary part of a retarded propagator) becomes

$$
\rho_{\phi}(\mathcal{P}) \equiv-\operatorname{Im}\left\{\left(p_{0}+i 0^{+}\right)^{2}-\epsilon_{\phi}^{2}\right\}^{-1}=\frac{\pi}{2 \epsilon_{\phi}}\left[\delta\left(p_{0}-\epsilon_{\phi}\right)-\delta\left(p_{0}+\epsilon_{\phi}\right)\right],
$$

where we denoted $\epsilon_{\phi}^{2} \equiv p^{2}+m_{\phi T}^{2}$. For the fermion propagator, recalling the self-energy $\Sigma_{\text {HTL }}$ within a massless plasma $[17,21]$, and taking subsequently the UR limit, we get

$$
\begin{aligned}
\phi_{\ell}(\mathcal{P}) & \equiv-\operatorname{Im}\left\{\not \mathcal{P}+\mathbb{Z}_{\mathrm{HTL}}\right\}_{p_{0} \rightarrow p_{0}+i 0^{+}}^{-1} \\
\left|p_{0}\right|>p & \frac{\pi \hbar_{+}}{2} \delta\left\{\left(p_{0}-p\right)(1+\tilde{L})-\frac{\delta m_{\ell T}^{2}}{2 p}\right\}+\frac{\pi \hbar_{-}}{2} \delta\left\{\left(p_{0}+p\right)(1-\tilde{L})+\frac{\delta m_{\ell T}^{2}}{2 p}\right\} \\
& \stackrel{\mathrm{UR}}{\approx} \frac{\pi}{2 \epsilon_{\ell}}\left[\overline{\mathcal{P}}_{\ell+} \delta\left(p_{0}-\epsilon_{\ell}\right)+\overline{\mathcal{P}}_{\ell-} \delta\left(p_{0}+\epsilon_{\ell}\right)\right],
\end{aligned}
$$

where $\tilde{L} \equiv \delta m_{\ell T}^{2} /\left(4 p^{2}\right) \ln \left\{\left(p_{0}+p\right) /\left(p_{0}-p\right)\right\}, \epsilon_{\ell}^{2} \equiv p^{2}+\delta m_{\ell T}^{2}$, and

$$
n_{ \pm} \equiv\left(1, \pm \mathbf{e}_{\mathbf{p}}\right), \quad \overline{\mathcal{P}}_{\ell \pm} \equiv \epsilon_{\ell} n_{\ell \pm}
$$

with $\mathbf{e}_{\mathbf{p}} \equiv \mathbf{p} / p$ standing for a unit vector. ${ }^{4}$ Dropping the subscript \pm implies that we consider the "particle" mode, i.e. $\overline{\mathcal{P}}_{\ell} \equiv \overline{\mathcal{P}}_{\ell+}$ and $n \equiv n_{+}$. For quarks and antiquarks, the spectral functions have the same form, just with the replacements $\delta m_{\ell T}^{2} \rightarrow m_{\infty}^{2}, \epsilon_{\ell} \rightarrow \epsilon_{q}, \epsilon_{\bar{q}}$.

Apart from propagators, HTL-resummed vertices are in principle needed as well. There is no correction to a Yukawa vertex, whereas the coupling of an electromagnetic current, of momentum $\mathcal{K}$, to a thermal quark-antiquark pair, takes place via $[22,23]$

$$
\Gamma^{\mu}=\gamma^{\mu}-\frac{m_{\infty}^{2}}{2} \int_{\mathbf{v}} \frac{\mathcal{V}^{\mu} \mathcal{V} \cdot \mathcal{P}_{q} \mathcal{V} \cdot \mathcal{P}_{\bar{q}}}{\mathcal{V}}, \quad \mathcal{V} \equiv(1, \mathbf{v})
$$

where the integral goes over the directions of the unit vector $\mathbf{v}$.

With these rules, we can compute the rates of interest. For right-handed neutrinos, picking up the particle branches that contribute to the $1 \rightarrow 2$ decay $\left(\equiv \rho_{\ell+}, \rho_{\phi+}\right)$, this gives

$$
\begin{aligned}
& \operatorname{Im}\left[\bar{u}_{\mathbf{k} \tau} a_{\mathrm{L}} \Pi_{a}^{\mathrm{R}} a_{\mathrm{R}} u_{\mathbf{k} \tau}\right]_{1 \rightarrow 2}^{\mathrm{HTL}}= \int_{\mathcal{P}_{\ell}, \mathcal{P}_{\phi}}(2 \pi)^{4} \delta^{(4)}\left(\mathcal{K}-\mathcal{P}_{\ell}-\mathcal{P}_{\phi}\right)\left[1-n_{\mathrm{F}}\left(\epsilon_{\ell}-\mu_{\ell}\right)+n_{\mathrm{B}}\left(\epsilon_{\phi}-\mu_{\phi}\right)\right] \\
& \times 4 \operatorname{Tr}\left\{\dot{\psi}^{\tau} a_{\mathrm{L}} \phi_{\ell+}\left(\mathcal{P}_{\ell}\right) a_{\mathrm{R}} \rho_{\phi+}\left(\mathcal{P}_{\phi}\right)\right\} \\
& \equiv \operatorname{scat}_{1 \rightarrow 2}(\ell, \phi) \Theta_{\mathrm{HTL}}^{\tau}\left(\mathcal{P}_{\ell}, \mathcal{P}_{\phi}\right) \\
& \Theta_{\mathrm{HTL}}^{\tau}\left(\mathcal{P}_{\ell}, \mathcal{P}_{\phi}\right) \stackrel{\mathrm{UR}}{\approx} 4 \mathcal{E}^{\tau} \cdot \overline{\mathcal{P}}_{\ell+},
\end{aligned}
$$

\footnotetext{
${ }^{4}$ The approximation on the last line of eq. (3.9) would not be justified if we considered processes very close to threshold, e.g. $M \sim m_{\phi T}+\delta m_{\ell T}$, so that the domain $\epsilon_{\ell} \sim \delta m_{\ell T}$ could give a substantial contribution.
} 
where $a_{\mathrm{L}(\mathrm{R})}=\left(1 \mp \gamma_{5}\right) / 2$, and the helicity projections read

$$
\mathcal{E}^{+} \equiv \frac{\omega+k}{2}\left(1, \mathbf{e}_{\mathbf{k}}\right), \quad \mathcal{E}^{-} \equiv \frac{\omega-k}{2}\left(1,-\mathbf{e}_{\mathbf{k}}\right) .
$$

Eqs. (3.14) and (3.15) give the analogues of eqs. (2.27) and (2.28), respectively, viz.

$$
\begin{aligned}
& \Theta_{\mathrm{HTL}}^{-} \underset{\mathrm{UR}}{\approx} \frac{2(\omega-k)\left(p_{\ell}+p_{\ell \|}\right) \epsilon_{\ell}}{p_{\ell}} \underset{\mathrm{UR}}{\approx} \frac{2 M^{2} \epsilon_{\ell}}{\omega}, \\
& \Theta_{\mathrm{HTL}}^{+} \underset{\mathrm{UR}}{\approx} \frac{2(\omega+k)\left(p_{\ell}-p_{\ell \|}\right) \epsilon_{\ell}}{p_{\ell}} \underset{\mathrm{UR}}{\approx} \frac{2 \omega p_{\perp}^{2}}{\epsilon_{\ell}} .
\end{aligned}
$$

For the UR limits, we made use of the fact that as far as energy-momentum conservation is concerned, eqs. (3.8) and (3.9) imply that the constraints take the same form as in vacuum,

$$
\omega=\epsilon_{\ell}+\epsilon_{\phi}, \quad \mathbf{k}=\mathbf{p}_{\ell}+\mathbf{p}_{\phi}, \quad k p_{\ell \|}=\omega \epsilon_{\ell}+\frac{m_{\phi T}^{2}-\delta m_{\ell T}^{2}-M^{2}}{2}
$$

and that therefore $p_{\ell} \stackrel{\mathrm{UR}}{\approx} p_{\ell \|} \stackrel{\mathrm{UR}}{\approx} \epsilon_{\ell}$.

For the electromagnetic current, mediated by the vertex from eq. (3.11), we similarly get

$$
\begin{aligned}
& \operatorname{Im}\left[\Pi^{\mathrm{R} \mu \nu}\right]_{1 \rightarrow 2}^{\mathrm{HTL}}= \int_{\mathcal{P}_{q}, \mathcal{P}_{\bar{q}}}(2 \pi)^{4} \delta^{(4)}\left(\mathcal{K}-\mathcal{P}_{q}-\mathcal{P}_{\bar{q}}\right)\left[1-n_{\mathrm{F}}\left(\epsilon_{q}-\mu_{q}\right)-n_{\mathrm{F}}\left(\epsilon_{\bar{q}}+\mu_{q}\right)\right] \\
& \times 2 N_{\mathrm{c}} \operatorname{Tr}\left\{\Gamma^{\mu} \phi_{q+}\left(\mathcal{P}_{q}\right) \Gamma^{\nu} \phi_{\bar{q}+}\left(\mathcal{P}_{\bar{q}}\right)\right\} \\
& \equiv \operatorname{scat}_{1 \rightarrow 2}(q, \bar{q}) \Theta_{\mathrm{HTL}}^{\mu \nu}\left(\mathcal{P}_{q}, \mathcal{P}_{\bar{q}}\right), \\
& \Theta_{\mathrm{HTL}}^{\mu \nu}\left(\mathcal{P}_{q}, \mathcal{P}_{\bar{q}}\right) \stackrel{\mathrm{UR}}{\approx} \frac{N_{\mathrm{c}}}{4}\left[\operatorname{Tr}\left(\Gamma^{\mu} \overline{\mathscr{P}}_{q}\right) \operatorname{Tr}\left(\Gamma^{\nu} \overline{\mathcal{P}}_{\bar{q}}\right)+\operatorname{Tr}\left(\Gamma^{\mu} \overline{\mathcal{P}}_{\bar{q}}\right) \operatorname{Tr}\left(\Gamma^{\nu} \overline{\mathscr{P}}_{q}\right)-4 \overline{\mathcal{P}}_{q} \cdot \overline{\mathcal{P}}_{\bar{q}} \operatorname{Tr}\left(\Gamma^{\mu} \Gamma^{\nu}\right)\right] .
\end{aligned}
$$

The influence of the vertex correction is tedious to work out. It can be shown, however, that the Ward identity $\mathcal{K}_{\mu} \Theta_{\mathrm{HTL}}^{\mu \nu}=0$ is satisfied within the UR approximation, and that for $\Theta_{\mathrm{HTL}}^{00}$ and $\Theta_{\text {HTL }}^{\mathrm{T}}$ the vertex corrections can be omitted [24], i.e. we can replace $\Gamma^{\mu} \rightarrow \gamma^{\mu}$. Thanks to the Ward identity we can use $\Theta_{\mathrm{HTL}}^{\mathrm{L}}=M^{2} \Theta_{\mathrm{HTL}}^{00} / k^{2}$. Then, employing energy-momentum conservation like in eq. (3.18), which implies $\mathbf{p}_{q} \cdot \mathbf{p}_{\bar{q}}=\epsilon_{q} \epsilon_{\bar{q}}+m_{\infty}^{2}-M^{2} / 2$, eq. (3.21) leads to

$$
\begin{aligned}
\Theta_{\mathrm{HTL}}^{\mathrm{L}} & \stackrel{\mathrm{UR}}{\approx} \frac{4 N_{\mathrm{c}} M^{2} \epsilon_{q} \epsilon_{\bar{q}}\left(2-n_{q} \cdot n_{\bar{q}}\right)}{k^{2}} \\
& =\frac{4 N_{\mathrm{c}} M^{2} \epsilon_{q} \epsilon_{\bar{q}}\left(p_{q} p_{\bar{q}}+\mathbf{p}_{q} \cdot \mathbf{p}_{\bar{q}}\right)}{p_{q} p_{\bar{q}} k^{2}} \stackrel{\mathrm{UR}}{\approx} \frac{8 N_{\mathrm{c}} M^{2} \epsilon_{q} \epsilon_{\bar{q}}}{\omega^{2}}, \\
\Theta_{\mathrm{HTL}}^{\mathrm{T}} \stackrel{\mathrm{UR}}{\approx} 8 N_{\mathrm{c}} \epsilon_{q} \epsilon_{\bar{q}}\left(n_{q \perp}^{i} n_{\bar{q} \perp}^{i}+n_{q} \cdot n_{\bar{q}}\right) & \\
& =\frac{8 N_{\mathrm{c}} \epsilon_{q} \epsilon_{\bar{q}}\left(p_{q} p_{\bar{q}}-p_{q \|} p_{\bar{q} \|}\right)}{p_{q} p_{\bar{q}}} \underset{\mathrm{UR}}{\approx} \frac{4 N_{\mathrm{c}} p_{\perp}^{2}\left(\epsilon_{q}^{2}+\epsilon_{\bar{q}}^{2}\right)}{\epsilon_{q} \epsilon_{\bar{q}}} .
\end{aligned}
$$

The last forms in eqs. (3.23) and (3.25) coincide with those in eq. (3.6), after multiplying with the factor $1 /\left(8 \epsilon_{q} \epsilon_{\bar{q}}\right)$ from the integration measure. 


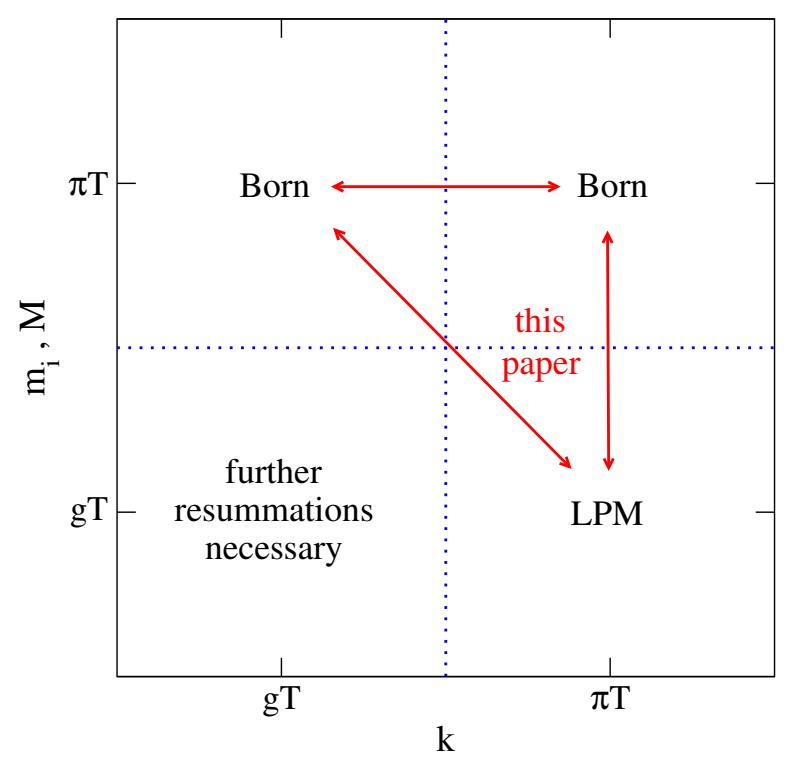

Figure 1. An overview of various momentum and mass domains, with $g \ll \pi$ denoting a generic coupling. The interpolation introduced in section 4 can be used in three of the four quadrants shown, but does not apply when all masses and momenta are very small (blindly extrapolating the interpolant into that quadrant, the result could even become negative, which is unphysical).

\section{Interpolation}

The results of sections 2 and 3 are parametrically correct in different mass and momentum domains, as illustrated in figure 1. The purpose of the present section is to suggest a smooth interpolation between these domains, making the results more broadly applicable.

The key idea is to generalize the Hamiltonians in eqs. (3.3) and (3.7) so that they apply beyond the UR limit. ${ }^{5}$ To this aim we keep the kinematic and mass structures from inside the Dirac- $\delta$ in eq. (2.25), and then just replace $p_{\perp}^{2} \rightarrow-\nabla_{\perp}^{2}$ and add the thermal width, viz.

$$
\begin{aligned}
\hat{H}_{\mathrm{inter}} \equiv & \frac{m_{a T}^{2}-\nabla_{\perp}^{2}+\frac{\left(m_{b}^{2}-m_{a}^{2}-M^{2}\right)^{2}}{4 k^{2}}}{2 \epsilon_{a}}+\frac{m_{b T}^{2}-\nabla_{\perp}^{2}+\frac{\left(m_{a}^{2}-m_{b}^{2}-M^{2}\right)^{2}}{4 k^{2}}}{2\left(\omega-\epsilon_{a}\right)}-\frac{\omega M^{2}}{2 k^{2}} \\
& -i \sum_{i} g_{\mathrm{E} i}^{2} C_{i} \phi\left(m_{\mathrm{E} i} \mathbf{y}_{\perp}\right) .
\end{aligned}
$$

Here $m_{a T}^{2} \equiv m_{a}^{2}+\delta m_{a T}^{2}$ and $m_{b T}^{2} \equiv m_{b}^{2}+\delta m_{b T}^{2}$ include thermal corrections, such that at high temperatures they go over to asymptotic thermal masses. The sum over $i$ goes over the gauge representations involved. Employing $m_{a T}^{2}$ and $m_{b T}^{2}$ also in the terms $\left(m_{b}^{2}-m_{a}^{2}-M^{2}\right)^{2} / k^{2}$ and $\left(m_{a}^{2}-m_{b}^{2}-M^{2}\right)^{2} / k^{2}$ would be formally a higher-order effect in the UR regime, nevertheless this procedure can be given a justification only under specific circumstances (cf. section 5), so for the moment we do not adopt such a recipe.

\footnotetext{
${ }^{5}$ We note in passing that in principle there could be several $(\equiv n)$ non-degenerate $1 \rightarrow 2$ channels for the non-equilibrium particle to decay into. Then $\hat{H}$ would become an $n \times n$-matrix, with kinetic terms appearing on the diagonal, whereas the width matrix takes a non-diagonal appearance in this basis [7].
} 
It is more subtle to generalize the second lines of eqs. (3.1) and (3.6) beyond the UR limit. After longitudinal momenta are eliminated through eq. (2.22), the matrix elements squared in eqs. (2.27), (2.28), (2.34), (2.35) are functions of energy variables, $p_{\perp}^{2}$, vacuum masses, and the variables $\omega, k, M^{2}$ that characterize the non-equilibrium particle. However, these are not independent of each other within the Born limit, since the Dirac- $\delta$ constraint in eq. (2.25) permits to eliminate $M^{2}$ in favour of $p_{\perp}^{2}$, or vice versa. The problem is that once thermal masses are introduced in eq. (4.1), previously equivalent representations are no longer so, and in fact even their IR sensitivities differ. Yet the correct IR structure is unique and needs to be present (cf. sections 3.2 and 6).

If the LPM expressions are known (cf. eqs. (3.1) and (3.6)), it is possible to make use of the freedom in order to choose a representation which goes over to the correct LPM one in the UR limit. Concretely, noting that terms without $p_{\perp}^{2}$ correspond to $\lim _{\mathbf{y}_{\perp} \rightarrow \mathbf{0}} \operatorname{Im}\left[g\left(\mathbf{y}_{\perp}\right)\right] / \pi$ and $p_{\perp}^{2}$ to $\lim _{\mathbf{y}_{\perp} \rightarrow \mathbf{0}} \operatorname{Im}\left[\nabla_{\perp} \cdot \mathbf{f}\left(\mathbf{y}_{\perp}\right)\right] / \pi$, and indicating this transition as

$$
\Theta\left(\mathcal{P}_{a}, \mathcal{K}-\mathcal{P}_{a}\right) \equiv \Theta\left(\epsilon_{a}, p_{\perp}^{2}\right) \rightarrow \widetilde{\Theta}\left(\epsilon_{a}, \mathbf{y}_{\perp}^{2}\right),
$$

we can identify ${ }^{6}$

$$
\begin{aligned}
& \widetilde{\Theta}^{-}=\frac{(\omega-k)\left[2(\omega+k) \epsilon_{\ell}+m_{\phi T}^{2}-M^{2}\right]}{k} \frac{\operatorname{Im}\left[g\left(\mathbf{y}_{\perp}\right)\right]}{\pi}, \\
& \widetilde{\Theta}^{+}=\frac{\left(m_{\phi T}^{2}-M^{2}\right)\left[2(\omega-k) \epsilon_{\ell}+m_{\phi T}^{2}-M^{2}\right]}{2 k \epsilon_{\ell}} \frac{\operatorname{Im}\left[g\left(\mathbf{y}_{\perp}\right)\right]}{\pi}+\frac{2 k}{\epsilon_{\ell}} \frac{\operatorname{Im}\left[\nabla_{\perp} \cdot \mathbf{f}\left(\mathbf{y}_{\perp}\right)\right]}{\pi}, \\
& \widetilde{\Theta}^{\mathrm{L}}=\frac{2 N_{\mathrm{c}} M^{2}\left[4 \epsilon_{q}\left(\omega-\epsilon_{q}\right)-M^{2}\right]}{k^{2}} \frac{\operatorname{Im}\left[g\left(\mathbf{y}_{\perp}\right)\right]}{\pi}, \\
& \widetilde{\Theta}^{\mathrm{T}}=4 N_{\mathrm{c}}\left\{\frac{4 k^{2} m_{q}^{2}+M^{4}}{4 \epsilon_{q}\left(\omega-\epsilon_{q}\right)} \frac{\operatorname{Im}\left[g\left(\mathbf{y}_{\perp}\right)\right]}{\pi}+\frac{\epsilon_{q}^{2}+\left(\omega-\epsilon_{q}\right)^{2}-M^{2}}{\epsilon_{q}\left(\omega-\epsilon_{q}\right)} \frac{\operatorname{Im}\left[\nabla_{\perp} \cdot \mathbf{f}\left(\mathbf{y}_{\perp}\right)\right]}{\pi}\right\} .
\end{aligned}
$$

A few important remarks are in order. First, we have replaced the Higgs vacuum mass by a Higgs thermal mass in eqs. (4.3) and (4.4), and do the same in the Born-level expressions; otherwise a symmetric phase computation would make no sense. Second, the denominators in eqs. (4.4) and (4.6) can have zeros. With the Born-level integration range incorporating finite masses, they would be avoided, but with a finite width, it is non-trivial to verify that there is no spectral weight in this domain. To be prudent, the integrations over $\epsilon_{\ell}$ or $\epsilon_{q}$ may be implemented as principal values. Third, the representations are not unique, as they could be modified by terms that are of higher order in the UR regime. It turns out there are consistency conditions between eq. (4.1) and eqs. (4.3)-(4.6) that need to be satisfied; we return to this in section 5. The specific forms in eqs. (4.1) and (4.3)-(4.6) have been chosen in order to guarantee a straightforward matching in section 6 , but we have

\footnotetext{
${ }^{6}$ For $\widetilde{\Theta}^{-}$, we start from eq. (2.27) and insert $p_{\ell \|}$ from eq. (2.22), with $m_{a} \rightarrow m_{\ell}=0$ and $m_{b} \rightarrow m_{\phi T}$. For $\widetilde{\Theta}^{+}$, we start from the first form in eq. (2.28), take $p_{\ell \|}$ from eq. (2.22), pull apart $2 M^{2}\left(\omega-\epsilon_{\ell}\right) / k$, and insert there $M^{2}$ from eq. (2.25). For $\widetilde{\Theta}^{\mathrm{L}}$, the form comes directly from eq. (2.34). For $\widetilde{\Theta}^{\mathrm{T}}$, we start from eq. (2.35), and insert $M^{2}$ from eq. (2.25), viz. $M^{2}=\left[k^{2}\left(p_{\perp}^{2}+m_{q}^{2}\right)+M^{4} / 4\right] /\left[\epsilon_{q}\left(\omega-\epsilon_{q}\right)\right]$.
} 
also successfully tested another implementation, ${ }^{7}$ which might instead facilitate a matching needed for an NLO computation in the UR regime.

Once the matrix elements / splitting functions have been fixed, the interpolation can be expressed as

$$
\Gamma_{1+n \leftrightarrow 2+n}^{\text {inter }}=\frac{\omega}{8 k} \int_{-\infty}^{\infty} \mathrm{d} \epsilon_{a}\left[1+n_{\sigma_{a}}\left(\epsilon_{a}-\mu_{a}\right)+n_{\sigma_{b}}\left(\omega-\epsilon_{a}-\mu_{b}\right)\right] \lim _{\mathbf{y}_{\perp} \rightarrow \mathbf{0}} \mathbb{P}\left\{\frac{\widetilde{\Theta}\left(\epsilon_{a}, \mathbf{y}_{\perp}^{2}\right)}{\epsilon_{a}\left(\omega-\epsilon_{a}\right)}\right\}
$$

with the wave functions solved from eq. (3.2) and the Hamiltonian taken from eq. (4.1). Such an interpolation on the integrand level smoothly connects the LPM-resummed and Born regimes. We stress, however, that the result is reliable only in the Born $\left(m_{i}, M \gg g T\right)$ and UR regimes $\left(m_{i}, M \ll k \sim \pi T\right)$, whereas it should not be used for $k, m_{i}, M \ll \pi T$ (cf. figure 1).

\section{Crosscheck of UV asymptotics}

According to figure 1, the results of the interpolation should go over to the Born ones at large masses. A special case is when the virtuality of the probe particle is made large, $M \gg m_{a}, m_{b}, \pi T$. The structure of thermal spectral functions is well understood in this limit [26], and merits a discussion.

We start by recalling the vacuum values of the interaction rates at the Born level:

$$
\begin{aligned}
& \left.\Gamma_{1 \leftrightarrow 2}^{\mathrm{Born}(-)}\right|_{T=0}=\left.\Gamma_{1 \leftrightarrow 2}^{\mathrm{Born}(+)}\right|_{T=0}=\quad \frac{\left(M^{2}+m_{\ell}^{2}-m_{\phi}^{2}\right) \sqrt{\lambda}\left(M^{2}, m_{\ell}^{2}, m_{\phi}^{2}\right)}{16 \pi M^{2}} \\
& M \gg m_{\phi}, m_{\ell}=0 \frac{1}{16 \pi}\left[M^{2}-2 m_{\phi}^{2}+\mathcal{O}\left(\frac{m_{\phi}^{4}}{M^{2}}\right)\right], \\
& \left.\Gamma_{1 \leftrightarrow 2}^{\mathrm{Born}(\mathrm{T})}\right|_{T=0}=\left.2 \Gamma_{1 \leftrightarrow 2}^{\mathrm{Born}(\mathrm{L})}\right|_{T=0}=\quad \frac{N_{\mathrm{c}}\left(M^{2}+2 m_{q}^{2}\right) \sqrt{\lambda}\left(M^{2}, m_{q}^{2}, m_{q}^{2}\right)}{6 \pi M^{2}} \\
& \stackrel{M \gg m_{q}}{=} \quad \frac{N_{\mathrm{c}}}{6 \pi}\left[M^{2}+\mathcal{O}\left(\frac{m_{q}^{4}}{M^{2}}\right)\right] .
\end{aligned}
$$

Now, if we include thermal mass corrections in the scalar mass, i.e. $m_{\phi}^{2} \rightarrow m_{\phi T}^{2}=m_{\phi}^{2}+\delta m_{\phi T}^{2}$, then eq. (5.1) suggests the presence of a correction of $\mathcal{O}\left(g^{2} T^{2}\right)$. To see the correct behaviour in this limit, requires a full NLO computation [27-29]. It turns out that there is a contribution proportional to $\mathcal{O}\left(\lambda T^{2}\right)$, however it is not equivalent to $\delta m_{\phi T}^{2}$. In the dilepton case, thermal corrections are of $\mathcal{O}\left(g^{2} T^{4} / M^{2}\right)[15,26]$, and again not related to $\delta m_{q T}^{4}$, even if the same power of $T$ would be obtained from eq. (5.2).

\footnotetext{
${ }^{7}$ For $\widetilde{\Theta}^{-}$, we start from the first form of eq. (3.16) and insert $p_{\ell \|}$ from eq. (3.18). For $\widetilde{\Theta}^{+}$, we start from the first form in eq. (3.17), take $p_{\ell \|}$ from eq. (3.18), pull apart $2 M^{2}\left(\omega-\epsilon_{\ell}\right) \epsilon_{\ell} /\left(k p_{\ell}\right)$, and insert there $M^{2}$ from eq. (2.25), with $m_{a} \rightarrow \delta m_{\ell T}$ and $m_{b} \rightarrow m_{\phi T}$. In both $\widetilde{\Theta}^{-}$and $\widetilde{\Theta}^{+}$, we then set $p_{\ell} \equiv \operatorname{sign}\left(\epsilon_{\ell}\right) \sqrt{\epsilon_{\ell}^{2}-\delta m_{\ell T}^{2}}$ and restrict the integration range in eq. (4.7) to $\left|\epsilon_{\ell}\right| \geq\left|\delta m_{\ell T}\right|$. We recall that for $\epsilon_{\ell} \sim \delta m_{\ell T}$, none of the approaches of this paper represents a correct description of soft physics, however this region gives a subleading contribution to eq. (4.7). An NLO computation in the UR regime would require the subtraction of the $\epsilon_{\ell} \sim \delta m_{\ell T}$ region in eq. (4.7), followed by its full-fledged HTL inclusion, as discussed in ref. [25] for the electromagnetic case.
} 
In any case, introducing thermal mass corrections in eq. (4.1), changes the asymptotics of $\Gamma_{1+n \leftrightarrow 2+n}^{\text {inter }}$. Generically, the changes are proportional to $\delta m_{a T}^{2}, \delta m_{b T}^{2}$, and such "offsets" are also visible in the numerical results of section 7 . As mentioned, these offsets do not agree with what a full NLO computation would yield, but there is no reason to worry, for the offsets are subleading corrections in the regime $M \gg g T$. In fact, they could be dealt with in connection with a matching, as described in section 6 .

It turns out, however, that the precise way in which thermal mass corrections are introduced in eq. (4.1), is very important. Without sufficient care, the UV asymptotics would display not only offsets, but even terms that grow with $M$, as we now demonstrate.

Considering the asymptotic regime, $M \gg \pi T \gg g^{2} T / \pi$, the width can be omitted in eq. (4.1). Physically, this means that we consider the $n=0$ version of eq. (4.7). Keeping separate handles for various appearances of masses, and undoing the transformation in eq. (4.2), the starting point can be written in a form similar to eq. (2.25), viz.

$$
\begin{aligned}
\Gamma_{1 \leftrightarrow 2}^{\text {inter }} \equiv & \int_{-\infty}^{+\infty} \mathrm{d} \epsilon_{a} \frac{\operatorname{sign}\left(\epsilon_{a}\left(\omega-\epsilon_{a}\right)\right)}{16 \pi k}\left[1+n_{\sigma_{a}}\left(\epsilon_{a}-\mu_{a}\right)+n_{\sigma_{b}}\left(\omega-\epsilon_{a}-\mu_{b}\right)\right] \int_{0}^{\infty} \mathrm{d} p_{\perp}^{2} \Theta\left(\epsilon_{a}, p_{\perp}^{2}\right) \\
& \times \delta\left\{p_{\perp}^{2}-\frac{2 \epsilon_{a}\left(\omega-\epsilon_{a}\right)}{\omega}\left[\frac{\omega M^{2}}{2 k^{2}}-\frac{m_{a T}^{2}+\frac{\left(m_{b}^{2}-m_{a}^{2}-M^{2}\right)^{2}}{4 k^{2}}}{2 \epsilon_{a}}-\frac{m_{b T}^{2}+\frac{\left(m_{a}^{2}-m_{b}^{2}-M^{2}\right)^{2}}{4 k^{2}}}{2\left(\omega-\epsilon_{a}\right)}\right]\right\} .
\end{aligned}
$$

Solving for the integration boundaries by requiring that the argument of the Dirac- $\delta$ is infinitesimally negative at $p_{\perp}^{2} \rightarrow 0$, eq. (2.20) gets generalized into

$$
\begin{aligned}
\epsilon_{a T}^{ \pm} & \equiv \frac{\omega\left(M^{2}+m_{a T}^{2}-m_{b T}^{2}\right)+\frac{M^{2} \Delta_{T}}{\omega} \pm k \sqrt{\lambda\left(M^{2}, m_{a T}^{2}, m_{b T}^{2}\right)-\left(\frac{M \Delta_{T}}{\omega}\right)^{2}}}{2 M^{2}}, \\
\Delta_{T} & \equiv m_{a}^{2}-m_{a T}^{2}+m_{b T}^{2}-m_{b}^{2} .
\end{aligned}
$$

For a fixed $k$ and masses, but taking $M$ large, this can be simplified into

$$
\epsilon_{a T}^{ \pm} \equiv \bar{\epsilon}_{a T} \pm \Delta \epsilon_{a T}, \quad \bar{\epsilon}_{a T} \stackrel{M \gg m_{i}, k}{\approx} \frac{M}{2}+\frac{m_{a}^{2}-m_{b}^{2}+k^{2} / 2}{2 M}, \quad \Delta \epsilon_{a T} \stackrel{M \gg m_{i}, k}{\approx} \frac{k}{2} .
$$

The important observation is that the average energy is determined by the masses $m_{a}^{2}, m_{b}^{2}$, appearing in the "subleading" mass corrections in eq. (5.3). The reason is that at $M \gg k$, these terms are enhanced by $M^{2} / k^{2}$ compared with the contribution from $m_{a T}^{2}, m_{b T}^{2}$.

Inserting now the matrix element from eq. (4.3), with $m_{\phi T}^{2} \rightarrow m_{b}^{2}$ and re-introducing $m_{a}^{2}$ as it appears in eq. (2.22), let us denote

$$
\epsilon_{0} \equiv \frac{M^{2}+m_{a}^{2}-m_{b}^{2}}{2(\omega+k)} \stackrel{M \gg m_{i}, k}{\approx} \frac{M-k}{2}+\frac{m_{a}^{2}-m_{b}^{2}+k^{2} / 2}{2 M} .
$$

Then we are faced with

$$
\Gamma_{1 \leftrightarrow 2}^{\operatorname{inter(-)}} \stackrel{M \gg m_{i}, k, \pi T}{\approx} \int_{\epsilon_{a T}^{-}}^{\epsilon_{a T}^{+}} \mathrm{d} \epsilon_{a} \frac{M^{2}\left(\epsilon_{a}-\epsilon_{0}\right)}{8 \pi k^{2}}=\frac{M^{2}\left(\epsilon_{a T}^{+}-\epsilon_{a T}^{-}\right)}{8 \pi k^{2}}\left(\frac{\epsilon_{a T}^{+}+\epsilon_{a T}^{-}}{2}-\epsilon_{0}\right) .
$$

Inserting eqs. (5.6) and (5.7), the leading term reproduces eq. (5.1). However, if the masses in the $\mathcal{O}(1 / M)$-parts did not match, the subleading correction would be of $\mathcal{O}(M)$. 
To summarize, in order to guarantee the presence of vacuum-like UV asymptotics [26], cf. eqs. (5.1), (5.2), it is essential to choose parameters such that the masses appearing in the "subleading" terms in the Hamiltonian, $\left(m_{b}^{2}-m_{a}^{2}-M^{2}\right)^{2} / k^{2}$ and $\left(m_{a}^{2}-m_{b}^{2}-M^{2}\right)^{2} / k^{2}$, match those appearing in the matrix elements squared, $\widetilde{\Theta}$. Hence, in the case of $\widetilde{\Theta}^{\mp}$ of eqs. (4.3) and (4.4), respectively, one should use $m_{a}^{2}=0, m_{b}^{2}=m_{\phi T}^{2}$ in the Hamiltonian of eq. (4.1). The dilepton Hamiltonian is instead unambiguous, given that $m_{q}^{2}-m_{\bar{q}}^{2}=m_{q T}^{2}-m_{\bar{q} T}^{2}=0$.

\section{Matching of IR divergences}

Apart from the $1+n \leftrightarrow 2+n$ scatterings that LPM resummation deals with, physics problems normally involve $2 \leftrightarrow 2$ and $1 \leftrightarrow 3$ scatterings as well as virtual corrections to $1 \leftrightarrow 2$ scatterings that cancel their IR divergences. However, the thermal part of the virtual corrections to $1 \leftrightarrow 2$ scatterings also includes the HTL effects that lead to the thermal masses appearing in the LPM resummation. These processes must not be counted twice, and therefore a subtraction is needed. Specifically, the subtraction should remove the most IR sensitive contributions, enhanced by $\sim g^{2} T^{2} / M^{2}$ over the Born-level result when $M \rightarrow 0$, from the virtual corrections to $1 \leftrightarrow 2$ scatterings, as these now appear as a part of LPM resummation, schematically as $\sim \sum_{n=0}^{\infty}\left(g^{2} T^{2} / M^{2}\right)^{n}$.

Depending on the formalism, the subtraction can be implemented either on the side of the virtual corrections to $1 \leftrightarrow 2$ scatterings [6], or on the side of LPM resummation [30]. The subtraction offers for a crosscheck of LPM resummation itself, verifying that IR sensitive effects that lead to a powerlike breakdown of the naive perturbative series are matched.

To implement the subtraction on the side of LPM result, we should "re-expand" the latter to $\mathcal{O}\left(g^{2} T^{2}\right)$. Given that the width in eqs. (3.3), (3.4) is of $\mathcal{O}\left(g^{4}\right)$ if we formally expand in $m_{\mathrm{E}}^{2}$, the only contribution at $\mathcal{O}\left(g^{2} T^{2}\right)$ comes from the thermal masses in eq. (4.1). Therefore, we can now focus on eq. (5.3), viz.

$$
\Gamma_{1 \leftrightarrow 2}^{\text {inter }}=\int_{\epsilon_{a T}^{-}}^{\epsilon_{a T}^{+}} \mathrm{d} \epsilon_{a} \frac{\operatorname{sign}\left(\epsilon_{a}\left(\omega-\epsilon_{a}\right)\right)}{16 \pi k}\left[1+n_{\sigma_{a}}\left(\epsilon_{a}-\mu_{a}\right)+n_{\sigma_{b}}\left(\omega-\epsilon_{a}-\mu_{b}\right)\right] \Theta\left[\epsilon_{a}, p_{\perp_{T}}^{2}\left(\epsilon_{a}\right)\right],
$$

where $p_{\perp T}^{2}\left(\epsilon_{a T}^{ \pm}\right)=0$ at the integration boundaries.

If we now set $m_{a T}^{2} \rightarrow m_{a}^{2}, m_{b T}^{2} \rightarrow m_{b}^{2}$, we recover the Born result:

$$
\left.\Gamma_{1 \leftrightarrow 2}^{\text {inter }}\right|^{\left(g^{0}\right)}=\Gamma_{1 \leftrightarrow 2}^{\text {Born }} .
$$

The first correction reads

$$
\left.\Gamma_{1 \leftrightarrow 2}^{\text {inter }}\right|^{\left(g^{2}\right)}=\delta m_{a T}^{2} \frac{\partial \Gamma_{1 \leftrightarrow 2}^{\text {inter }}}{\partial m_{a T}^{2}}+\delta m_{b T}^{2} \frac{\partial \Gamma_{1 \leftrightarrow 2}^{\text {inter }}}{\partial m_{b T}^{2}} .
$$

Straightforward differentiation yields

$$
\begin{aligned}
\frac{\partial \Gamma_{1 \leftrightarrow 2}^{\text {inter }}}{\partial m_{a T}^{2}}= & \frac{\operatorname{sign}\left(\epsilon_{a}^{+}\left(\omega-\epsilon_{a}^{+}\right)\right)}{16 \pi k}\left[1+n_{\sigma_{a}}\left(\epsilon_{a}^{+}-\mu_{a}\right)+n_{\sigma_{b}}\left(\omega-\epsilon_{a}^{+}-\mu_{b}\right)\right] \Theta\left(\epsilon_{a}^{+}, 0\right) \frac{\partial \epsilon_{a T}^{+}}{\partial m_{a T}^{2}} \\
& -\frac{\operatorname{sign}\left(\epsilon_{a}^{-}\left(\omega-\epsilon_{a}^{-}\right)\right)}{16 \pi k}\left[1+n_{\sigma_{a}}\left(\epsilon_{a}^{-}-\mu_{a}\right)+n_{\sigma_{b}}\left(\omega-\epsilon_{a}^{-}-\mu_{b}\right)\right] \Theta\left(\epsilon_{a}^{-}, 0\right) \frac{\partial \epsilon_{a T}^{-}}{\partial m_{a T}^{2}} \\
& +\int_{\epsilon_{a}^{-}}^{\epsilon_{a}^{+}} \mathrm{d} \epsilon_{a} \frac{\operatorname{sign}\left(\epsilon_{a}\left(\omega-\epsilon_{a}\right)\right)}{16 \pi k}\left[1+n_{\sigma_{a}}\left(\epsilon_{a}-\mu_{a}\right)+n_{\sigma_{b}}\left(\omega-\epsilon_{a}-\mu_{b}\right)\right] \frac{\partial \Theta\left(\epsilon_{a}, p_{\perp}^{2}\right)}{\partial p_{\perp}^{2}} \frac{\partial p_{\perp T}^{2}}{\partial m_{a T}^{2}},
\end{aligned}
$$


and similarly for $\partial \Gamma_{1 \leftrightarrow 2}^{\mathrm{inter}} / \partial m_{b T}^{2}$. The derivatives originate from the Dirac- $\delta$ in eq. (5.3),

$$
\begin{array}{rlrl}
\frac{\partial \epsilon_{a T}^{ \pm}}{\partial m_{a T}^{2}} & =\mp \frac{k^{2}\left(\omega-\epsilon_{a}^{ \pm}\right)}{\omega M^{2}\left(\epsilon_{a}^{+}-\epsilon_{a}^{-}\right)}, & \frac{\partial \epsilon_{a T}^{ \pm}}{\partial m_{b T}^{2}} & =\mp \frac{k^{2} \epsilon_{a}^{ \pm}}{\omega M^{2}\left(\epsilon_{a}^{+}-\epsilon_{a}^{-}\right)}, \\
\frac{\partial p_{\perp T}^{2}}{\partial m_{a T}^{2}}=-\frac{\omega-\epsilon_{a}}{\omega}, & \frac{\partial p_{\perp T}^{2}}{\partial m_{b T}^{2}}=-\frac{\epsilon_{a}}{\omega} .
\end{array}
$$

Now, going to the UR limit, the integration boundaries from eq. (2.20) take the values $\epsilon_{a}^{ \pm} \stackrel{\mathrm{UR}}{\longrightarrow}\{-\infty, 0, \omega,+\infty\}$. IR divergences could therefore originate from inverse powers of $\epsilon_{a}^{ \pm}$ or $\omega-\epsilon_{a}^{ \pm}$on the first two rows of eq. (6.4), or from inverse powers of $\epsilon_{a}$ or $\omega-\epsilon_{a}$ on the third row of eq. (6.4). From eq. (6.5), we see that no inverse powers originate from the boundary terms, however the division by $M^{2}$ shows that contributions $\mathcal{O}\left(\delta m_{a T}^{2} / M^{2}\right), \mathcal{O}\left(\delta m_{b T}^{2} / M^{2}\right)$ do appear. In eq. (6.6), we see no inverse powers either. However, virtual corrections do contain inverse powers that take the form of the last line of eq. (6.4). Therefore, it is essential to have the correct representation for $\Theta$, such that $\partial_{p_{\perp}^{2}} \Theta\left(\epsilon_{a}, p_{\perp}^{2}\right)$ reproduces these IR divergences.

Let us illustrate the matching of IR divergences for the case of right-handed neutrinos first. A method to determine virtual corrections was worked out in ref. [31]. The starting point is to determine the real $1 \rightarrow 3$ rate, given in its eq. (2.13). Identifying the poles and residues of the matrix element squared, the virtual corrections can be identified, given in eq. (2.37) of ref. [31]. The virtual corrections contain thermal 1-loop integrals of various types, of which only a subclass can lead to a HTL contribution, proportional to $T^{2}$ [32]. Among those in eq. (2.37) of ref. [31], the only one is the mixed fermion-boson loop, weighted by a loop momentum, denoted by $B\left(\mathcal{P}_{\tilde{\ell}} ; \ell, \gamma\right) \mathcal{E} \cdot \mathcal{P}_{\ell}$. Specifically, carrying out the angular integral in eq. (2.29) of ref. [31] and taking the UR limit, yields $(\tilde{\mathcal{P}}=(\tilde{\epsilon}, \tilde{\mathbf{p}}), \tilde{p}=|\tilde{\mathbf{p}}|)$

$$
\begin{aligned}
B(\tilde{\mathcal{P}} ; a, b)\left(\alpha \mathcal{P}_{a}+\beta \mathcal{P}_{b}\right) & \\
\stackrel{\mathrm{UR}}{\longrightarrow} & \left(0, \frac{\tilde{\mathbf{p}}}{\tilde{p}^{2}}\right) \frac{\alpha-\beta}{8 \pi^{2}} \int_{0}^{\infty} \mathrm{d} \epsilon \epsilon\left[n_{\sigma_{a}}\left(\epsilon-\mu_{a}\right)+n_{\sigma_{a}}\left(\epsilon+\mu_{a}\right)-n_{\sigma_{b}}\left(\epsilon-\mu_{b}\right)-n_{\sigma_{b}}\left(\epsilon+\mu_{b}\right)\right] \\
& +\left(1, \frac{\tilde{\mathbf{p}} \tilde{\epsilon}}{\tilde{p}^{2}}\right) \mathcal{O}\left(\frac{T^{2}, \mu_{i}^{2}}{\tilde{p}}\right) .
\end{aligned}
$$

If one of the particles is a fermion and the other is a boson, the thermal distributions add up $\left(n_{-}=-n_{\mathrm{F}}\right.$ according to eq. (2.8)), and we recover a thermal fermion mass squared, notably

$$
\frac{1}{8 \pi^{2}} \int_{0}^{\infty} \mathrm{d} \epsilon \epsilon\left[n_{\mathrm{F}}\left(\epsilon-\mu_{a}\right)+n_{\mathrm{F}}\left(\epsilon+\mu_{a}\right)+2 n_{\mathrm{B}}(\epsilon)\right]=\frac{1}{16}\left(T^{2}+\frac{\mu_{a}^{2}}{\pi^{2}}\right) .
$$

Applying eqs. (6.7), (6.8) to the appropriate term in eq. (2.37) of ref. [31], we find

$$
\begin{aligned}
\Delta \Gamma_{1 \leftrightarrow 2}^{\mathrm{Born}(\tau)} & \supset-2\left(g_{1}^{2}+3 g_{2}^{2}\right) \operatorname{scat}_{1 \leftrightarrow 2}(\tilde{\ell}, \phi) B\left(\mathcal{P}_{\tilde{\ell}} ; \ell, \gamma\right) \mathcal{E}^{\tau} \cdot \mathcal{P}_{\ell} \\
& \stackrel{\mathrm{UR}}{\approx} 2 \operatorname{scat}_{1 \leftrightarrow 2}(\tilde{\ell}, \phi) \mathcal{E}^{\tau} \cdot\left[\frac{\delta m_{\ell T}^{2}}{\epsilon_{\tilde{\ell}}}\left(0, \frac{\mathbf{p}_{\tilde{\ell}}}{\epsilon_{\tilde{\ell}}}\right)+\mathcal{O}\left(\frac{g^{2} T^{2}, g^{2} \mu_{\ell}^{2}}{\epsilon_{\tilde{\ell}}}\right)\left(1, \frac{\mathbf{p}_{\tilde{\ell}}}{\epsilon_{\tilde{\ell}}}\right)\right] .
\end{aligned}
$$

Here $\tilde{\ell}$ labels an on-shell lepton, distinguished from the $\ell$ inside the loop $B$, whereas the vectors $\mathcal{E}^{\tau}$ are from eq. (3.15). We note that $\mathcal{E}^{-}$is of $\mathcal{O}\left(M^{2} / \omega\right)$ in the UR limit, whereby 
$\Delta \Gamma_{1 \leftrightarrow 2}^{\mathrm{Born}(-)}$ is of $\mathcal{O}\left(g^{4} T^{2}\right)$ and beyond our resolution. In contrast, $\mathcal{E}^{+} \stackrel{\mathrm{UR}}{\approx}(\omega, \mathbf{k})$ is of $\mathcal{O}(\omega)$. Recalling from eq. (2.22) that $\mathbf{k} \cdot \mathbf{p}_{\tilde{\ell}} \underset{\mathrm{UR}}{\approx} \omega \epsilon_{\tilde{\ell}}$, the 2 nd term in the square brackets in eq. (6.10) drops out for $\tau=+$. In total, then,

$$
\Delta \Gamma_{1 \leftrightarrow 2}^{\mathrm{Born}(+)} \stackrel{\mathrm{UR}}{\supset}-2 \delta m_{\ell T}^{2} \operatorname{scat}_{1 \leftrightarrow 2}(\tilde{\ell}, \phi) \frac{\omega}{\epsilon_{\tilde{\ell}}} .
$$

This shows an IR divergence (inverse power of $\epsilon_{\tilde{\ell}}$ ) of the type that appears on the last line

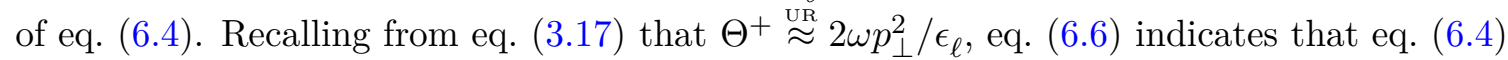
exactly matches eq. (6.11) at $\epsilon_{\tilde{\ell}} \rightarrow 0$, if we have set $m_{a T}^{2}=\delta m_{\ell_{T}}^{2}$.

The same exercise can be carried out for the dilepton case. In the normalization employed in eq. (2.29), the would-be $1 \rightarrow 3$ contribution to the interaction rate reads

$$
\begin{aligned}
\Gamma_{1 \rightarrow 3}^{\mathrm{Born}(\mu \nu)}= & \operatorname{scat}_{1 \rightarrow 3}(q, g, \bar{q}) 8 g_{3}^{2} C_{3} N_{\mathrm{c}}\left\{\eta^{\mu \nu}\right. \\
& +\frac{\eta^{\mu \nu}\left(s_{q \bar{q}}+M^{2}\right)-2\left(\mathcal{P}_{q}^{\mu} \mathcal{K}^{\nu}+\mathcal{P}_{q}^{\nu} \mathcal{K}^{\mu}\right)}{2\left(s_{q g}-m_{q}^{2}\right)}+\frac{\eta^{\mu \nu}\left(s_{q \bar{q}}+M^{2}\right)-2\left(\mathcal{P}_{\bar{q}}^{\mu} \mathcal{K}^{\nu}+\mathcal{P}_{\bar{q}}^{\nu} \mathcal{K}^{\mu}\right)}{2\left(s_{\bar{q} g}-m_{q}^{2}\right)} \\
& +\frac{m_{q}^{2}\left[\eta^{\mu \nu} M^{2}-2\left(\mathcal{P}_{\bar{q}}^{\mu} \mathcal{K}^{\nu}+\mathcal{P}_{\bar{q}}^{\nu} \mathcal{K}^{\mu}\right)+4 \mathcal{P}_{\bar{q}}^{\mu} \mathcal{P}_{\bar{q}}^{\nu}\right]}{\left(s_{q g}-m_{q}^{2}\right)^{2}} \\
& +\frac{m_{q}^{2}\left[\eta^{\mu \nu} M^{2}-2\left(\mathcal{P}_{q}^{\mu} \mathcal{K}^{\nu}+\mathcal{P}_{q}^{\nu} \mathcal{K}^{\mu}\right)+4 \mathcal{P}_{q}^{\mu} \mathcal{P}_{q}^{\nu}\right]}{\left(s_{\bar{q} g}-m_{q}^{2}\right)^{2}} \\
& \left.+\frac{\left(2 m_{q}^{2}-M^{2}\right)\left[\eta^{\mu \nu} M^{2}-\left(\mathcal{P}_{g}^{\mu} \mathcal{K}^{\nu}+\mathcal{P}_{g}^{\nu} \mathcal{K}^{\mu}\right)-2\left(\mathcal{P}_{q}^{\mu} \mathcal{P}_{\bar{q}}^{\nu}+\mathcal{P}_{q}^{\nu} \mathcal{P}_{\bar{q}}^{\mu}\right)\right]-2 M^{2} \mathcal{P}_{g}^{\mu} \mathcal{P}_{g}^{\nu}}{\left(s_{q g}-m_{q}^{2}\right)\left(s_{\bar{q} g}-m_{q}^{2}\right)}\right\}
\end{aligned}
$$

Here $g$ denotes a gluon, $s_{a b} \equiv\left(\mathcal{P}_{a}+\mathcal{P}_{b}\right)^{2}$, and scat ${ }_{1 \rightarrow 3}$ is defined in eqs. (2.2)-(2.4) of ref. [31].

Like above, virtual corrections can be deduced from the poles of eq. (6.12), and only first-order poles lead to terms involving HTLs in the UR limit. It is helpful to consider the transverse and vector projections, from eqs. (2.31) and (2.33). Both yield the same HTLs, which after writing $s_{q \bar{q}}=\left(\mathcal{K}-\mathcal{P}_{g}\right)^{2}$ gives an expression analogous to eq. (6.9), viz.

$$
\Delta \Gamma_{1 \leftrightarrow 2}^{\mathrm{Born}(\mathrm{T}, \mathrm{V})} \supset 16 g_{3}^{2} C_{3} N_{\mathrm{c}}\left[\operatorname{scat}_{1 \leftrightarrow 2}(\tilde{q}, \bar{q}) B\left(\mathcal{P}_{\tilde{q}} ; q, g\right)+\operatorname{scat}_{1 \leftrightarrow 2}(q, \tilde{\bar{q}}) B\left(\mathcal{P}_{\tilde{q}} ; \bar{q}, g\right)\right] \mathcal{K} \cdot \mathcal{P}_{g} .
$$

Given that $\mathrm{T}$ and $\mathrm{V}$ yield the same IR divergence, there is none in the $\mathrm{L}$ channel.

In the UR limit, eq. (6.13) can be approximated like in eq. (6.10). Again the 2nd term in the square brackets drops out, when we make use of $\mathbf{k} \cdot \mathbf{p}_{\tilde{q}} \underset{\text { UR }}{\approx} \omega \epsilon_{\tilde{q}}$ and $\mathbf{k} \cdot \mathbf{p}_{\tilde{q}} \underset{\text { UR }}{\approx} \omega \epsilon_{\tilde{q}}$. This leads to the analogue of eq. (6.11), viz.

$$
\Delta \Gamma_{1 \leftrightarrow 2}^{\mathrm{Born}(\mathrm{T}, \mathrm{V})} \stackrel{\mathrm{UR}}{\supset}-4 N_{\mathrm{c}} m_{\infty}^{2}\left[\operatorname{scat}_{1 \leftrightarrow 2}(\tilde{q}, \bar{q}) \frac{\omega}{\epsilon_{\tilde{q}}}+\operatorname{scat}_{1 \leftrightarrow 2}(q, \tilde{\bar{q}}) \frac{\omega}{\epsilon_{\tilde{q}}}\right] .
$$

Let us compare eq. (6.14) with eq. (6.4). From eq. (2.35) and from the projection of eq. (2.29) according to eq. (2.33), we have

$$
\begin{aligned}
& \Gamma_{1 \leftrightarrow 2}^{\mathrm{Born}(\mathrm{T})}=\operatorname{scat}_{1 \leftrightarrow 2}(q, \bar{q}) 4 N_{\mathrm{c}}\left(-2 p_{\perp}^{2}+M^{2}\right), \\
& \Gamma_{1 \leftrightarrow 2}^{\mathrm{Born}(\mathrm{v})}=\operatorname{scat}_{1 \leftrightarrow 2}(q, \bar{q}) 4 N_{\mathrm{c}}\left(2 m_{q}^{2}+M^{2}\right) .
\end{aligned}
$$



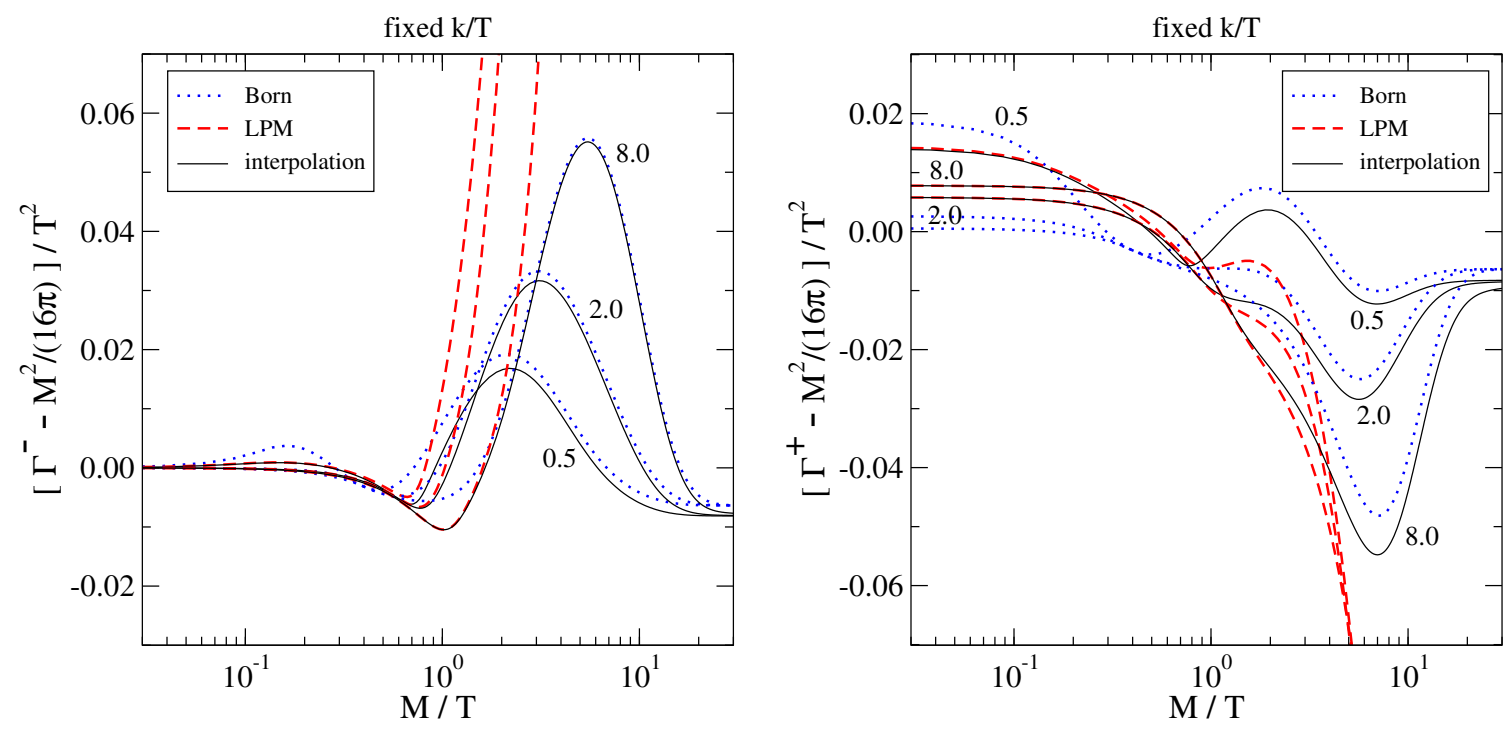

Figure 2. Results for the right-handed neutrino interaction rates in the $\tau=-$ (left) and $\tau=+$ (right) channels, after the subtraction of the leading-order vacuum contributions at $M \gg m_{\phi T}$, cf. eq. (5.1). The value of $k / T$ has been indicated next to the curves.

As discussed below eq. (6.6), $p_{\perp}^{2}$ as such does not lead to an IR divergence. But if we eliminate $M^{2}$ in favour of $p_{\perp}^{2}$ by making use of eq. (2.25), as has been done in order to arrive at eq. (4.6), then the corresponding contributions depend on the thermal mass correction as

$$
\frac{\partial M^{2}}{\partial m_{\infty}^{2}} \equiv \frac{\partial M^{2}}{\partial p_{\perp}^{2}}\left(\frac{\partial p_{\perp T}^{2}}{\partial m_{q T}^{2}}+\frac{\partial p_{\perp T}^{2}}{\partial m_{\bar{q} T}^{2}}\right) \stackrel{\mathrm{UR}}{\approx} \frac{\omega^{2}}{\epsilon_{q} \epsilon_{\bar{q}}}\left(-\frac{\epsilon_{\bar{q}}}{\omega}-\frac{\epsilon_{q}}{\omega}\right)=-\left(\frac{\omega}{\epsilon_{q}}+\frac{\omega}{\epsilon_{\bar{q}}}\right) .
$$

Thereby eq. (6.14) is indeed reproduced. Incidentally, this is the same divergence that renders the strict NLO expression for the vector spectral function logarithmically divergent and discontinuous across the light cone [15].

To summarize this section, we have verified that after a proper choice of variables in the matrix elements squared, the LPM resummed results match the IR divergences that appear in virtual corrections to the Born result. If an LPM resummed result is combined with a computation which already includes virtual corrections to $1 \leftrightarrow 2$ scatterings, eqs. (6.2)-(6.4) should be subtracted, in order to avoid double counting.

\section{Numerical evaluation}

In this section, we illustrate the recipe of section 4 numerically. In figure 2, results based on $\Gamma_{1 \leftrightarrow 2}^{\text {Born }}, \Gamma_{1+n \leftrightarrow 2+n}^{\mathrm{LPM}}, \Gamma_{1+n \leftrightarrow 2+n}^{\mathrm{inter}}$, are shown for right-handed neutrinos of either helicity, with matrix elements squared taken from eqs. (4.3) and (4.4). As we are in the symmetric phase, the lepton vacuum mass has been omitted and the scalar vacuum mass replaced by its thermal counterpart $\left(m_{\ell}=0, m_{\phi} \rightarrow m_{\phi T}\right)$; the thermal masses have been set to $\delta m_{\ell T}=0.3 T, m_{\phi T}=0.4 T, m_{\mathrm{E} 1}=0.5 T, m_{\mathrm{E} 2}=0.9 T$; and the couplings to $g_{1}=1 / 3$, $g_{2}=2 / 3$. A few separate values of $k / T$ have been chosen, with the results plotted as a 

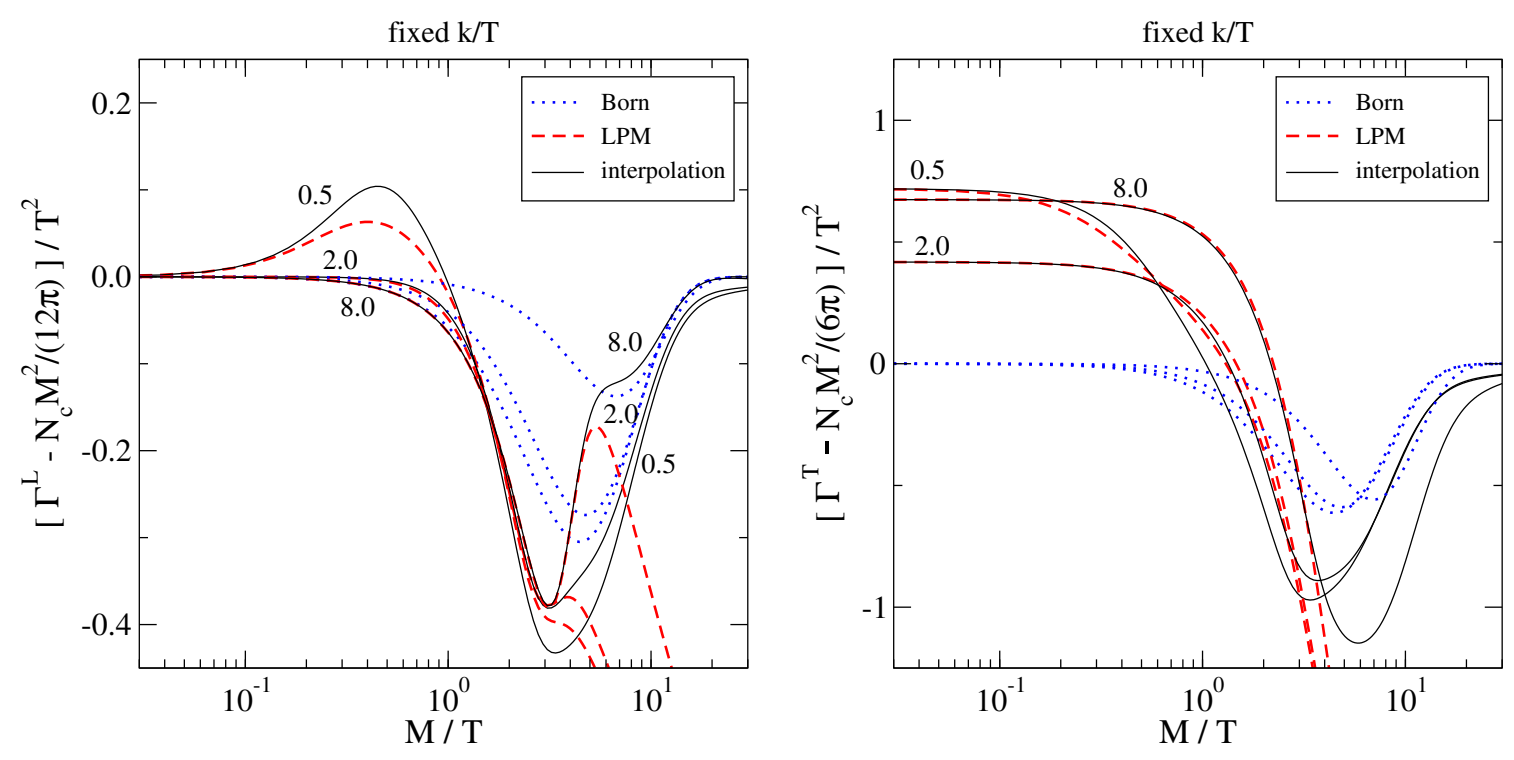

Figure 3. Results for the dilepton interaction rates in the $\mathrm{L}$ (left) and $\mathrm{T}$ (right) channels, after the subtraction of the leading-order vacuum contributions, cf. eq. (5.2). The value of $k / T$ has been indicated next to the curves.

function of $M / T$. The same exercise is repeated for both channels of the vector correlator in figure 3, with matrix elements squared from eqs. (4.5) and (4.6), setting quark vacuum masses to zero $\left(m_{q}=0\right)$, thermal masses to $m_{\infty}=1.0 T, m_{\mathrm{E} 3}=2.2 T$, and the gauge coupling to $\alpha_{\mathrm{s}}=0.3$. For a better plotting resolution, leading-order vacuum contributions that would otherwise dominate at large $M / T$ (cf. section 5), have been subtracted in both systems.

The conclusion from figures 2 and 3 is that the interpolations indeed agree with LPM results for $M \ll \pi T$, and with the shape of the Born results for $M \gg \pi T$, however in the latter regime we observe constant offsets, cf. section 5. It is appropriate to stress that the offsets are only visible because we have subtracted the dominant terms, and that the offsets originate from fermionic thermal mass corrections which are absent in the Born results, and could be subtracted through a matching computation, as described in section 6 .

\section{Conclusions and outlook}

At leading non-trivial order in the weak-coupling expansion, general thermal interaction rates consist of two classes of contributions. On one hand, there are $2 \leftrightarrow 2$ and the crossed $1 \leftrightarrow 3$ processes as well as virtual corrections to $1 \leftrightarrow 2$ processes whose role is to cancel IR divergences. These have recently been analysed in some generality in ref. [31]. On the other hand, there are real $1+n \leftrightarrow 2+n$ processes, with $n \geq 0$, that need often to be summed to all orders in $n$, through LPM resummation [1-4]. The latter set constitutes the focus of the present paper. Even though the two classes do not completely decouple from each other, in the sense that specific thermal corrections appearing in the virtual corrections to $1 \leftrightarrow 2$ processes also play a role in LPM resummation, whereby a subtraction procedure is needed 
for evading double counting (cf. section 6), for practical purposes the kinematics and the theoretical tools playing a role in the two sets are very different. Therefore the methods of ref. [31] are of little help here, and a dedicated analysis has become necessary.

Concretely, we have generalized the light-cone Hamiltonian that plays a role in LPM resummation, beyond the ultrarelativistic regime for which it was originally derived. Our key result is eq. (4.1), which sets the Hamiltonian in a form that respects the correct kinematics of the Born limit as well (cf. eq. (2.25)).

Apart from the Hamiltonian, matrix elements squared, or splitting functions, play a role in the formalism. Even though they can in principle be derived at Born level, the Born results can be put in various forms, by making use of energy-momentum conservation. Yet only specific representations reproduce the correct IR divergences when the Hamiltonian is modified by thermal corrections. We have shown two separate ways to verify that the variables chosen in eqs. (4.3)-(4.6) are consistent from this point of view, going either through an explicit HTL computation (cf. section 3.2), or through the inspection of the IR divergences that appear in NLO virtual corrections to $1 \leftrightarrow 2$ processes (cf. section 6 ).

Another consistency check on the interpolation is obtained by inspecting the UV domain, in which the mass of the probe particle is made large. We have verified that our formalism produces a qualitatively correct behaviour in this limit (cf. section 5). For this, appearances of thermal masses need to be chosen consistently in eq. (4.1) and in eqs. (4.3)-(4.6).

Despite being able to cover a broad kinematic regime with our interpolation, we stress that there is a particular corner, namely that where momenta and masses are all small compared with the temperature, in which neither Born nor LPM results are reliable (cf. figure 1). Consequently our interpolation does not work in the vicinity of this domain. It would be interesting to remedy this shortcoming, however this represents a demanding task, due to the complicated resummations required.

The computations that we have carried out apply formally at leading order in the weak-coupling expansion, i.e. $\mathcal{O}\left(g^{2} T^{2}\right)$ in the ultrarelativistic regime. A relevant question is to what extent known or unknown NLO corrections, suppressed only by $\mathcal{O}(g)$ [20], could be incorporated in our setup. This would be straightforward for the objects appearing in eq. (4.1), notably asymptotic masses [33,34] or the thermal width [35-38]. Furthermore, in the dilepton case, an analytic continuation of our framework permits for the determination of certain correlation lenghts, which may be compared with lattice simulations, to test empirically the influence of NLO corrections [39]. That said, there are also NLO effects whose inclusion requires a more significant effort, as they involve regions where thermal fermions (active leptons or quarks in our examples) become soft. To treat these regions correctly, full-fledged HTL computations are required, generalizing on the ultrarelativistic approximation discussed in section 3.2. In addition, subtractions beyond those discussed in section 6 are needed, in order to avoid double counting the soft-fermion region at first order in the width [25].

\section{Acknowledgments}

J.G. acknowledges support by the Région Pays de la Loire under a PULSAR grant. M.L. was supported by the Swiss National Science Foundation (SNF), through grant 200020B-188712. 
Open Access. This article is distributed under the terms of the Creative Commons Attribution License (CC-BY 4.0), which permits any use, distribution and reproduction in any medium, provided the original author(s) and source are credited.

\section{References}

[1] P. Aurenche, F. Gelis and H. Zaraket, Landau-Pomeranchuk-Migdal effect in thermal field theory, Phys. Rev. D 62 (2000) 096012 [hep-ph/0003326] [INSPIRE].

[2] P.B. Arnold, G.D. Moore and L.G. Yaffe, Photon emission from ultrarelativistic plasmas, JHEP 11 (2001) 057 [hep-ph/0109064] [INSPIRE].

[3] P. Aurenche, F. Gelis, G.D. Moore and H. Zaraket, Landau-Pomeranchuk-Migdal resummation for dilepton production, JHEP 12 (2002) 006 [hep-ph/0211036] [INSPIRE].

[4] A. Anisimov, D. Besak and D. Bödeker, Thermal production of relativistic Majorana neutrinos: Strong enhancement by multiple soft scattering, JCAP 03 (2011) 042 [arXiv: 1012.3784] [INSPIRE].

[5] B. Garbrecht, F. Glowna and P. Schwaller, Scattering rates for leptogenesis: Damping of lepton flavour coherence and production of singlet neutrinos, Nucl. Phys. B 877 (2013) 1 [arXiv: 1303.5498] [INSPIRE].

[6] I. Ghisoiu and M. Laine, Right-handed neutrino production rate at T>160 GeV, JCAP 12 (2014) 032 [arXiv: 1411.1765] [INSPIRE].

[7] J. Ghiglieri and M. Laine, Neutrino dynamics below the electroweak crossover, JCAP 07 (2016) 015 [arXiv: 1605.07720] [INSPIRE].

[8] P. Hernández, M. Kekic, J. López-Pavón, J. Racker and J. Salvado, Testable baryogenesis in seesaw models, JHEP 08 (2016) 157 [arXiv:1606.06719] [INSPIRE].

[9] J. Ghiglieri and M. Laine, Precision study of GeV-scale resonant leptogenesis, JHEP 02 (2019) 014 [arXiv: 1811.01971] [INSPIRE].

[10] D. Bödeker and D. Schröder, Equilibration of right-handed electrons, JCAP 05 (2019) 010 [arXiv: 1902.07220] [INSPIRE].

[11] S. Biondini and J. Ghiglieri, Freeze-in produced dark matter in the ultra-relativistic regime, JCAP 03 (2021) 075 [arXiv: 2012.09083] [INSPIRE].

[12] J. Klarić, M. Shaposhnikov and I. Timiryasov, Reconciling resonant leptogenesis and baryogenesis via neutrino oscillations, Phys. Rev. D 104 (2021) 055010 [arXiv:2103.16545] [INSPIRE].

[13] M. Drees and B. Najjari, Energy spectrum of thermalizing high energy decay products in the early universe, JCAP 10 (2021) 009 [arXiv:2105.01935] [INSPIRE].

[14] J. Ghiglieri and G.D. Moore, Low mass thermal dilepton production at NLO in a weakly coupled quark-gluon plasma, JHEP 12 (2014) 029 [arXiv:1410.4203] [INSPIRE].

[15] G. Jackson and M. Laine, Testing thermal photon and dilepton rates, JHEP 11 (2019) 144 [arXiv: 1910.09567] [INSPIRE].

[16] J. Ghiglieri and M. Laine, GeV-scale hot sterile neutrino oscillations: a derivation of evolution equations, JHEP 05 (2017) 132 [arXiv:1703.06087] [INSPIRE]. 
[17] H.A. Weldon, Effective fermion masses of order gT in high-temperature gauge theories with exact chiral invariance, Phys. Rev. D 26 (1982) 2789 [INSPIRE].

[18] M.E. Carrington, Effective potential at finite temperature in the Standard Model, Phys. Rev. D 45 (1992) 2933 [INSPIRE].

[19] P. Aurenche, F. Gelis and H. Zaraket, A simple sum rule for the thermal gluon spectral function and applications, JHEP 05 (2002) 043 [hep-ph/0204146] [INSPIRE].

[20] S. Caron-Huot, O(g) plasma effects in jet quenching, Phys. Rev. D 79 (2009) 065039 [arXiv:0811.1603] [INSPIRE].

[21] V.V. Klimov, Collective Excitations in a Hot Quark Gluon Plasma, Sov. Phys. JETP 55 (1982) 199 [Zh. Eksp. Teor. Fiz. 82 (1982) 336] [INSPIRE].

[22] J. Frenkel and J.C. Taylor, Hard thermal QCD, forward scattering and effective actions, Nucl. Phys. B 374 (1992) 156 [InSPIRE].

[23] E. Braaten and R.D. Pisarski, Simple effective Lagrangian for hard thermal loops, Phys. Rev. D 45 (1992) R1827 [INSPIRE].

[24] P. Aurenche, F. Gelis and H. Zaraket, Enhanced thermal production of hard dileptons by $3 \rightarrow 2$ processes, JHEP 07 (2002) 063 [hep-ph/0204145] [INSPIRE].

[25] J. Ghiglieri, J. Hong, A. Kurkela, E. Lu, G.D. Moore and D. Teaney, Next-to-leading order thermal photon production in a weakly coupled quark-gluon plasma, JHEP 05 (2013) 010 [arXiv: 1302.5970] [INSPIRE].

[26] S. Caron-Huot, Asymptotics of thermal spectral functions, Phys. Rev. D 79 (2009) 125009 [arXiv: 0903.3958] [INSPIRE].

[27] A. Salvio, P. Lodone and A. Strumia, Towards leptogenesis at NLO: the right-handed neutrino interaction rate, JHEP 08 (2011) 116 [arXiv:1106.2814] [INSPIRE].

[28] M. Laine and Y. Schröder, Thermal right-handed neutrino production rate in the non-relativistic regime, JHEP 02 (2012) 068 [arXiv:1112.1205] [INSPIRE].

[29] S. Biondini, N. Brambilla, M.A. Escobedo and A. Vairo, An effective field theory for non-relativistic Majorana neutrinos, JHEP 12 (2013) 028 [arXiv:1307.7680] [INSPIRE].

[30] I. Ghisoiu and M. Laine, Interpolation of hard and soft dilepton rates, JHEP 10 (2014) 083 [arXiv: 1407.7955] [INSPIRE].

[31] G. Jackson and M. Laine, Efficient numerical integration of thermal interaction rates, JHEP 09 (2021) 125 [arXiv:2107.07132] [INSPIRE].

[32] E. Braaten and R.D. Pisarski, Soft amplitudes in hot gauge theories: A general analysis, Nucl. Phys. B 337 (1990) 569 [INSPIRE].

[33] S. Caron-Huot, On supersymmetry at finite temperature, Phys. Rev. D 79 (2009) 125002 [arXiv:0808.0155] [INSPIRE].

[34] G.D. Moore and N. Schlusser, The nonperturbative contribution to asymptotic masses, Phys. Rev. D 102 (2020) 094512 [arXiv:2009.06614] [InSPIRE].

[35] M. Panero, K. Rummukainen and A. Schäfer, Lattice Study of the Jet Quenching Parameter, Phys. Rev. Lett. 112 (2014) 162001 [arXiv:1307.5850] [INSPIRE].

[36] J. Ghiglieri and H. Kim, Transverse momentum broadening and collinear radiation at NLO in the $\mathcal{N}=4$ SYM plasma, JHEP 12 (2018) 049 [arXiv: 1809.01349] [INSPIRE]. 
[37] G. Jackson and M. Laine, A thermal neutrino interaction rate at NLO, Nucl. Phys. B 950 (2020) 114870 [arXiv: 1910.12880] [inSPIRE].

[38] G.D. Moore, S. Schlichting, N. Schlusser and I. Soudi, Non-perturbative determination of collisional broadening and medium induced radiation in QCD plasmas, JHEP 10 (2021) 059 [arXiv:2105.01679] [INSPIRE].

[39] B.B. Brandt, A. Francis, M. Laine and H.B. Meyer, A relation between screening masses and real-time rates, JHEP 05 (2014) 117 [arXiv:1404.2404] [INSPIRE]. 